\title{
II. Technik und Fetisch
}

\section{Einleitung}

\section{Handlung und Vorgeschichte}

Wenn hier der Plot und die Vorgeschichte von Über Raben (2002) referiert werden, muss sofort eingeräumt werden, dass es sich dabei nur um eine, wenn auch wahrscheinliche und belegbare, so doch nie restlos gesicherte Variante dessen handelt, was im Roman selbst bzw. noch vor Einsetzen der Romanhandlung geschieht. Der Grund für diese Unsicherheit, die sich auch in zahlreichen Rezeptionszeugnissen spiegelt, ${ }^{1}$ liegt in der abweisend sglatten $<$ Oberfläche dieser Narration, einer bruchund lückenlosen Erzählweise, die nur unter Berücksichtigung kleinster Indizien Rückschlüsse auf die Vorgeschichte zulässt.

Erzählt wird in zwei getrennten Handlungssträngen in alternierenden Kapiteln, einmal in der ersten, einmal in der dritten Person. Beide Handlungsstränge sind in ihrer Perspektive eng beschränkt auf die Wahrnehmungen und aktuellen Gedankengänge des Protagonisten bzw. der Protagonistin. Vordergründig erfährt man so von zwei voneinander vermeintlich unabhängigen Handlungsverläufen, die einmal einen Mann im Nationalpark Gesäuse beim Klettern, einmal die Schulwoche einer Dreizehnjährigen an einem Wiener Innenstadtgymnasium zeigen. Während der Mann sich in schwindelnder Höhe (in einer der klassischen Kletterrouten des Gesäuses, der Rosskuppe-Dachl-Verschneidung) in einer Felshöhle einnistet, durchlebt im Gegenzug dazu das Mädchen, Valentina, einen äußerst geregelten, ruhigen Alltag - geht zur Schule, erledigt Einkäufe, leistet einer behinderten Nachbarin Gesellschaft.

Beide Handlungsstränge strotzen vor Wahrnehmungsdetails - Mode und Ausrüstung, Landschaft und Alltag, verschiedene Verrichtungen im Gebirge und in der Stadt; nichts davon beantwortet die in der Lektüre auftretenden und bald einigermaßen drängenden Fragen: Warum führt der Mann ein Gewehr mit sich, warum gebirge (2002); Tauber, Überwindung einer hohen Emotions-Kletterwand (2002); u.v.m. 
beobachtet er durch das Zielfernrohr akribisch die Umgebung, und warum rechnet er mit einer Schar von Verfolgern? Was sind das für seltsame handwerkliche Verrichtungen, denen das Mädchen da nachgeht, warum treten ihre Eltern über den Zeitraum einer Woche nie in Erscheinung?

Weil die Rätselstruktur des Textes schließlich doch auf ein Verbrechen hindeutet, ${ }^{2}$ steht Über Raben in einem Verhältnis zur Gattung des Kriminalromans. Weil jedoch Detektion, Detektiv und Leichnam - also die kriminalliterarische Indizienlektüre, ihr zentraler Akteur und ihr initiales Zeichen - fehlen, ist dieses Verhältnis keines der Zugehörigkeit, stattdessen eines der metafiktionalen (und, wenn man so will, postmodernen) Bezugnahme. ${ }^{3}$ Die lückenhafte Narration steht hier im Dienst dieser Bezugnahme, insofern sie die kriminalliterarische Indizienlektüre provoziert - und zugleich keine Festlegung auf eine endgültige Version jener Ereignisse zulässt, die dem Einsetzen der Handlung vorangehen.

Es gibt eine Vielzahl von Anhaltspunkten zur Rekonstruktion der Vorgeschichte, die, wie anzunehmen ist, das Movens beider Handlungsstränge darstellt. Jedoch erfahren sämtliche Details eine völlige erzählökonomische Gleichbehandlung, unabhängig von ihrer möglichen Funktion für eine Indizienlektüre: Keines ist wichtiger als das andere. Bekleidung erhält genau so viel Aufmerksamkeit wie die Gebrauchsanweisung eines Insektensprays, das Gewehr so viel wie das Gipfelbuch.

Indem beide Erzählstränge zugleich diese Überfülle und massive Leerstellen aufweisen, provozieren sie eine zwangsläufig überforderte Lektüre; dennoch lässt sich der Mann in den Bergen bald als der in Valentinas Schule krankgeschriebene Lehrer Schneider (Deutsch und Turnen) identifizieren. Zudem rücken weitere Handlungselemente in den Fokus: dass Valentina etwa einen Teil der Wohnung durch Öffnen der Fenster kühlt (es ist tiefster Winter) oder regelmäßig seltsame Einkäufe tätigt (Holzkitt, Insektenvernichter, Raumspray); dass Schneider neben all dem alpinistischen Equipment und dem Gewehr auch Hausübungen von Valentina mit sich führt, die er zudem »ziemlich auswendig« (ÜR 166) kennt, und, auch das wird verdächtig, dass seine Patronenschachtel nicht mehr ganz voll ist (ÜR 160).

Diese Hausübungen bzw. die aus ihnen gebrachten Zitate können alle im Roman disparaten Aspekte in einer Lesart zusammenführen:

Der dritte Aufsatz. >Mein Weihnachtsabend s. Eine Tanne wird gekauft, die bis an die Decke reicht. Der Christbaumschmuck wird ergänzt, denn jedes Jahr geht etHeinzl stellt etwa die Vermutung auf, in der Wohnung des Mädchens könnten sich die Leichen der Eltern befinden. Vgl.: Polt-Heinzl, Höhenlager und Abgründe (2004).

3 Zu diesem Verhältnis zwischen Über Raben und dem Kriminalroman vgl. ausführlicher der Verf., Das sah ergenau (2013); zum postmodernen Kriminalroman und zum fehlenden Körper vgl. Plochocki, Body, Letter, and Voice (2010), v.a. Kap. II. 
was zu Bruch. Man bereitet ein Essen mit teurem Fisch und jungen Erbsen zu und hintennach einem Kastanienpudding. Schließlich werden Lieder gespielt, >)ingle Bells<, >Feliz Navidad « und am Schluss `Stille Nacht‘. Dann die Glocke. >lch weiß, dass alle da sind. Ich sehe sie nicht, aber ich weiß es. Man schiebt mich ins Zimmer. Ich rieche den Baum und die Wunderkerzen. Ich stehe da, mit geschlossenen Augen, diese große rote Schleife um den Bauch. Irgendjemand kichert leise, und jemand anderer sagt: Frohe Weihnachten.८ (ÜR 234)

Diese lückenhafte Szene könnte man sich so vorstellen: Ein Kind feiert Weihnachten, es trägt ein Kleid mit roter Schleife, die Augen hält es aus Vorfreude und zur Steigerung der Spannung geschlossen. Diese Lesart enthält allerdings Ungereimtheiten: Das Kichern, die zwei offenbar unbekannten Personen (»Irgendjemand«) evozieren eine Bedrohung, und von da aus wird auch die Schleife um den Bauch suspekt: Das Kind, so lautet eine andere, plausiblere Lesart, ist in diesem Weihnachtsfest das Geschenk, die Augen sind aus Angst geschlossen. ${ }^{4}$ Dann würde Valentinas Aufsatz Missbrauchserlebnisse darstellen und gäbe nachträglich dem gesamten Text einen Hintergrund, vor dem auch andere Passagen aus den Hausübungen verständlich werden - etwa die Schlussbemerkung nach dem Aufsatz über eine Landschulwoche: "Die meisten fuhren nach diesen anstrengenden Tagen gern wieder nach Hause. Manche nicht. (ÜR 233) Auch Bemerkungen aus den Kapiteln des Mädchens (die Aufsätze kommen nur von Seiten des Lehrers zur Sprache) lassen sich so im Nachhinein einordnen, etwa die gedankliche Erwiderung auf die Aussage einer Lehrerin, als Mädchen müsse man froh sein, keine Essstörung zu haben: »Als Mädchen muss man ab und zu über ganz andere Dinge froh sein.« (ÜR 120)

Die rätselhaften täglichen Verrichtungen Valentinas in den verschlossenen Räumen ihrer Wohnung sowie ihr Bemühen, diese Zimmer kühl zu halten, geben einen Hinweis auf den Grund für Schneiders Flucht in die Berge: Ihre ritualhaften Gänge mit Insektenvertilger, Raumspray und später Fixativ sowie das Öffnen der Fenster (ÜR 101, 144f., 222, 227 u.ö.) legen Verwesungsprozesse nahe, ihre Anwendung von Holzkitt (ÜR 50 u.ö.), die auf den unschuldigen Tipp einer Klassenkollegin zurückgeht (»Womit stopft man kleine Löcher?<, frage ich Simone. >Welche Löcher? « Simple kleine runde Löcher.« - ÜR 40), das Verschließen von Einschusslöchern - nach dieser Lesart: von Schusswunden. Schneider wäre demnach geflohen, weil er Valentinas Hausübungen als die Anzeige lang anhaltenden Missbrauchs gelesen und daraufhin ihre Eltern erschossen hat - ein Vorgehen, das in seiner Drastik womöglich auf Schneiders mangelndes Vertrauen

4 Vgl. Hochgatterers kurze Erzählung Weg vom Krippenspiel (2007), darin den ganz ähnlichen Bericht eines Mädchens. 
in rechtmäßige Wege der Ahndung hinweist und auf die Schwierigkeit der Beweisfindung im Inneren der hermetischen Institution Familie (eine Problemlage, die Hochgatterer mehrfach verhandelt). ${ }^{5}$ Seine Tat wiederum versucht nun Valentina zu verheimlichen, indem sie die toten Körper konserviert.

Der psychologische Notstand, dass der Kern eines Traumas nicht versprachlicht werden kann, wird, indem die toten Eltern sowie ihre Ermordung keine Erwähnung finden, zur Voraussetzung der Narration. Valentinas Konservierungsmaßnahmen, zugleich Vertuschung wie Umsorgung der Leichen, sistieren den kriminalliterarisch üblichen Ablauf, der in eine Untersuchung und schließlich in eine Auflösung münden würde, und eröffnen das Zeitfenster von einer Woche, deren Zeuge man beim Lesen wird; die Arbeit der Detektion bleibt inzwischen der Lektüre überlassen.

Der dramaturgischen Notwendigkeit folgend, ist das Ende des Romans in Valentinas Fall offen: Der Ferienbeginn und das drohende Tauwetter stellen ganz neue Voraussetzungen für ihre Vertuschungsaktion dar. Im Fall Schneiders hingegen drängt die Dramaturgie des Selbstjustiz-Dramas in Richtung eines definitiveren Endes: Er stürzt, in einem Moment geistiger Umnachtung, aus der Höhle in den Tod und bringt damit die Reihe der Gewalttaten im Roman zu einem unwiderruflichen Abschluss.

Die in dieser Lesart rekonstruierten Geschehnisse liegen zwar der Handlung des Textes voraus; als Ursache, die nachträglich zahlreiche Details der Textoberfläche als Indizien lesbar macht, werden sie aber erst spät im Text erkennbar: Der wichtigste Fingerzeig, das obenstehende Zitat aus der Hausübung zum Thema >Weihnachtsabend des Mädchens darüber, was sie im Kunstunterricht basteln könnte (»am ehesten zwei Köpfe«, ÜR 42, oder Dominosteine mit »Doppelnullen«, ÜR 4of., 48), werden im Nachhinein ebenso vielsagend wie das Referat, das sie halten möchte (»über Haut und Knochen. Oder so in der Art zumindest«, ÜR 86) und die Musik, die sie gerade hört (Georg Friedrich Händels Alexanderfest von 1736, ebenfalls ein Text mit Rachemotiv). ${ }^{6}$

5 Siehe z.B. den gewalttätigen Familienvater in Die Süße des Lebens, die Missbrauchssituationen in Das Matratzenhaus und in Caretta Caretta; vgl. auch den kurzen Text Das Milchhautkind (2005).

6 In diesem Intertext bewegt der Barde Timotheus seinen Herrn Alexander den Großen nach gewonnener Schlacht dazu, die feindliche Stadt aus Rache niederzubrennen, womit sich in der Sangeskunst des Barden die Texte des Mädchens in ihren Folgen - und vielleicht ihren Motiven - spiegeln. Die Passage, die sie über mehrere Tage hin mit Hilfe eines Wörterbuches übersetzt (ÜR 77, 101, 122, 125, 127, 130, 182, 188, 197, 221f.), gibt den zentralen Ruf nach Rache wieder; an zwei vielsagenden Textstellen hat die Übersetzerin dabei Schwierigkeiten: »Revenge, revenge, Timotheus cries «, wohingegen Valentina hier »time of the cries « hört (ÜR 197) -»See the furies arise, / See the snakes that they rear, / How they hiss in their hair, / And the 


\section{Eine Umwelt aus Dingen}

Als Skandalon des Textes sind Missbrauch und Selbstjustiz - in perfider Umkehrung der Dringlichkeit - erst auf den zweiten Blick auszumachen. Was hingegen sofort augenscheinlich wird, ist die Übermacht der Dinge in der Wahrnehmung der beiden zentralen Figuren: Beide bewegen sich in einer Welt aus Markenartikeln, beide registrieren detailliert die Embleme ihrer Umgebung, lassen ihre Gestimmtheit sowie ihr Handeln von Artefakten beeinflussen.

Die Wahrnehmungen Valentinas zur Bekleidung ihrer MitschülerInnen und LehrerInnen addieren sich $\mathrm{zu}$ seitenlangen Beschreibungen von und Kommentaren zu Modeartikeln (dazu siehe ausführlich Kap. III). Die aktuelle Gegenwart Schneiders wiederum setzt sich für diesen vor allem aus Ausrüstungsgegenständen zusammen, deren Erscheinung und Funktionieren er wachsam registriert. Er bemerkt das verdächtige Geräusch, das sein Schistock von sich gibt (ÜR 10, 17), den gerissenen Kabelzug an einem Schischuh (ÜR 10), die schwergängige Bindung (ÜR 56); allein die Packlisten, die sich aus dem tranchenweisen Transport seiner Ausrüstung vom Auto über eine Hütte und den Wandfuß in die Höhle ergeben, füllen ein paar Seiten (ÜR 21, 59, 61, 154, 201 u.ö.). Das ist hinsichtlich seiner Situation - isoliert von anderen Menschen, umgeben nur von unbelebter Natur und Ausrüstung - wenig verwunderlich. Aber auch in seinen Erinnerungen spielen neben den Menschen die Dinge prägnante Rollen: In der Erinnerung an hochalpinen Geschlechtsverkehr mit seiner Exfrau Elvira findet ein Jack-Wolfskin-Shirt besondere Erwähnung (ÜR 21), in einer Szene im Büro des Schuldirektors ein Resopalmöbel (ÜR 66), Erinnerungen an Bergtouren handeln von verwaisten Rucksäcken (ÜR 116f.) oder verlorengehenden Stirnbändern (ÜR 231). Die Dinge stehen im Vordergrund: möglicherweise in puncto Handlungsrelevanz, sicher jedoch hinsichtlich ihrer emotionalen Bedeutung. Anlässlich einer Packung Neapolitaner-Waffeln werden neben ernährungsphysiologischen auch ganz andere Gesichtspunkte relevant: "Niemandem hatte er jemals vom Geschmack der Waffeln und von der wunderbaren roten Schrift auf dem durchsichtigen Zellophan erzählt, niemandem, Elvira nicht, den Kollegen nicht und den Schülern schon gar nicht.« (ÜR 56)

sparkles that flash from their eyes! / Behold a ghastly band, / Each a torch in his hand! / Those are Grecian ghosts, that in battle were slain, / And unbury'd, remain / Inglorious on the plain« - wobei wiederum »unburied « ein Wort ist, das Valentina auch mit Hilfe des Wörterbuchs nicht ohne weiteres übersetzen kann (ÜR 222). Alternativ zur minutiösen Indizienlektüre führt damit auch der Intertext des Liedes auf die Spur zum Plot shinter dem Plotı, dem Rachedrama, das der Handlung von Über Raben in dieser Lesart vorausgeht. Die fehlgehörten Passagen erschweren die Lektüre dieses Hinweises - um dann jedoch gerade mit den Passagen, die sich der Übersetzung vorerst verweigern, eine Kürzest-Inhaltsangabe des Textes zu liefern: stime of the cries«, eine Zeit des Weinens, in Gegenwart der Unbegrabenen. Vgl. zur Indizienlektüre in Über Raben der Verf., Das sah er genau (2013). 
Für eine antifetischistische Lektüre bietet der Text somit eine Unzahl von Ansatzpunkten. An die oben gebrachten Beispiele und Aufzählungen schließen diejenigen Dinge, die in einem engen Zusammenhang mit dem Mord stehen, nahtlos an, wenn sie sich nicht gar überlappen. Markennamen treten nicht nur in Zusammenhang mit Modeartikeln auf, sondern auch bei Raumspray und Insektenvernichter, dasselbe gilt für das Gewehr. Die allgegenwärtige Marke homogenisiert die Dinge unabhängig von ihrer Handlungsrelevanz über ein und dieselbe warenästhetische Perspektive, was dazu beiträgt, die Indizienlektüre zu erschweren.

Gewehr, Insektenspray etc. stehen an der Schnittstelle von warenästhetischer bzw. ausrüstungstechnischer Überfülle auf der einen und kriminalistischem (bzw. kriminalliterarischem) Handlungszusammenhang auf der anderen Seite. Die Dinge haben nicht nur Fetischcharakter (so alltäglich dieser sein mag), sie stehen auch in Verbindung mit der psychiatrischen bzw. kriminellen Devianz der handelnden

Personen. Ein Zusammenhang, wie er zwischen Verbrechen und Tatwaffe herrscht, wäre nun auch anderswo zu vermuten: In welchem Verhältnis stehen also die vielfältigen Fetischismen im Text zum Verbrechen?

\section{Fetischismus am Berg}

\section{Die Berge - "wir lieben sie hassend und bekämpfend"}

Schon dem landläufigen Verständnis nach ist Alpinismus eine Art Fetischdienst. Aber auch der Blick ins Archiv ${ }^{7}$ legt weitreichende Kongruenzen zwischen alpinis-

7 Ich beschränke mich für das hier zu durchforstende Archiv auf das Alpenvereinsjahrbuch (mit ein paar Ausnahmen hinsichtlich relevanter Artikel in anderen Organen) und einzelne maßgebliche Buchpublikationen, darunter eine frühe Geschichte des österreichischen Alpinismus von Gustav Gröger u. Josef Rabl sowie zwei streitbare und daher besonders ergiebige Titel von Karl Ziak und Eugen Guido Lammer. Den Hinweis auf letztere verdanke ich dem auf ein breites Textkorpus aufbauenden Aufsatz von Stefan Kaufmann. Ebenfalls auf ein breites alpinistisches Textkorpus gegründet und damit ausgesprochen hilfreich sind die (m.W. erste) Psychologie des Bergsteigens von Ulrich Aufmuth und die Kulturgeschichte des Bergsteigens von Dagmar Cünther (Aufmuth nimmt dabei eine Doppelstellung ein, indem er den betreffenden Diskurs, anstatt ihn bloß zu analysieren, auch selbst fortschreibt). Historische Grundlagen entnehme ich über die genannten Quellen hinaus Peter Crupps Ceschichte des Alpinismus. Vgl.: Zeitschrift des Deutschen und Österreichischen Alpenvereins [alternativ: »Berg«, »Alpenvereinsjahrbuch «] (im Folgenden unter Angabe des Jahres zitiert als »ZDÖAV«); Crögeru. Rabl, Die Entwicklung der Hochtouristik in den österreichischen Alpen (1890); Lammer, Wie anders ist das Besteigen der Alpen geworden! (1937); Ziak, Der Mensch und die Berge (1936); Kaufmann, Technik am Berg (2006); Aufmuth, Zur Psychologie des Bergsteigens (1988 [1984]); Cünther, Alpine Quergänge (1998); Grupp, Faszination Berg (2008). 
tischen und fetischtheoretischen Texten nahe. Dies soll hier in aller Kürze an den großen Themenfeldern des Fetischdiskurses gezeigt werden.

Schon das alpinistische Beharren auf dem Irrationalen, dieses »wider besseres Wissen «, das den Sport ausmacht, weist in diese Richtung. ${ }^{8}$ Offensichtlich ist zudem (mehr noch als beim Fliegenfischen), wie sehr im alpinistischen Sprechen Phantasmen des >Natürlichen zungsphantasie eine Rolle spielen. Es ist vor allem das Begriffspaar >Ganzheit $<$ und >Zerstückelungく, das im alpinistischen Kontext fetischistische Konstellationen aufscheinen lässt. Ende des 19. Jahrhunderts stellen etwa die Autoren eines historischen Werks zum Alpinismus fest, dass der eigentlich homogene Berg für einen Kletterer, der die (sportliche) Felstour der Eistour vorzieht, in Fragmente zerfalle; »der Ersteiger hat es mit dem Kamin, dem Bande, der Platte zu thun, der Berg selbst verliert dabei seine Individualität, und keck tritt ihm der Besieger auf's Haupt. ${ }^{9}$ Diese >Epigonen seien ein "gottloses Geschlecht«, sei doch die Einheit des Berges eine sakrosankte. - Entsprechend wird das Bergerlebnis regelmäßig mittels religiöser Metaphorik ins Bild gesetzt; ${ }^{10}$ im Archiv des Alpinismus hat man es durchwegs mit einer Frage des rechten Glaubens zu tun. Die Trennlinie zwischen wahrem und falschem Glauben ist, ganz wie im Diskurs um den Fetisch der Afrikaner, die zwischen Ganzheit und Zerstückelung: Dem Bild einer natürlichen Ganzheit des Berges steht das einer künstlichen, frevelhaften Fragmentierung gegenüber; dem erhabenen Schauer die respektlose Zurichtung des Gottes.

In den 1930ern bringt zudem der bekannte Alpinist und Autor Karl Ziak (auf den weiter unten noch eingegangen werden wird) das Aufkommen des sportlichen schwierigkeitsalpinismus (bei dem nicht der Gipfel, sondern der Anspruch der gewählten Route im Vordergrund steht) mit dem Warencharakter in Zusammenhang. "So wie die Industrie sich damals nicht mehr begnügte, nur das Notwendige zu schaffen, sondern auch das Überflüssige, den Luxus erzeugte, so begnügten sich die Bergsteiger nun nicht mehr, den Gipfel überhaupt zu erreichen. « ${ }^{11}$ Die Spaltung in das notwendige und das bloß differenzielle Merkmal der Ware findet hier ein alpinistisches Pendant, wenn das Ornament der Routenwahl (»das Überflüssige, [der] Luxus«) anstelle des bloßen Gipfels (dem »Notwendigen«) in den Vordergrund rückt.

Auch die Ankunft der konkreten Ware im Gebirge lässt die Idiosynkrasien des Diskurses hervortreten. Die Autoren des Alpenvereinsjahrbuchs etwa betreiben Ende der 1980er, Anfang der 1990er Jahre auf besorgte bis ironische Weise Konsumkri-

8 Vgl. Aufmuth, Zur Psychologie des Bergsteigens (1988 [1984]), S. 11f.

9 Gröger u. Rabl, Die Entwicklung der Hochtouristik in den österreichischen Alpen (1890), S. 225; Karl Ziak zitiert diese Passage (vgl. Ziak, Der Mensch und die Berge (1936), S. 177).

10 Vgl. etwa die Nachweise in Grupp, Faszination Berg (2008), S. 273.

11 Ziak, Der Mensch und die Berge (1936), S. 126. 
tik. ${ }^{12}$ Unterschieden wird zwischen zwei verschiedenen Arten der Dingbeziehung, zum bewährten wie zum modischen Ding, mit entsprechenden Werthaltungen:

Es ist nun mal nicht anders: Der Bergsteiger lebt mit den Dingen, die er gebraucht und benützt und die ihm oftmals das Leben erhalten. Er will möglichst lange mit ihnen leben, denn sie bedeuten ihm viel. Er gehört nicht zu denen, die wegwerfen, was ihnen nicht mehr gefällt. Er ist einer, der behält, selbst, wenn es nicht mehr gefällt. Altes ist für ihn ein Ehrentitel, denn es beweist gute Bewährung, und es erinnert ihn an Momente des Clücks und der Freude. ${ }^{13}$

Es ist so naheliegend wie irrational, anzunehmen, dass die Dinge sviel bedeuten<, wo sie doch hauptsächlich funktionieren sollen, und dass die Abnutzung der Dinge auf irgendeine Weise die Bewährung, ihr Alter die Ehre des Menschen belegen könnte. Warenästhetik trifft auf Bergsteigeraskese: In restloser Übereinstimmung mit den zahllosen Grenzziehungsmanövern, die mittels des Fetischbegriffs historisch vorgenommen wurden, wird hier zwischen einem wahrhaftigen und einem verblendeten Irrationalismus unterschieden, wobei letzterer unter das Fetisch-Verdikt fällt und ersterer als Norm installiert wird.

Es sind die klassischen Fetischismen der Antifetischisten, die sich in den Diskussionen zu Sport und Warenästhetik am Berg finden. Wendet man sich hingegen von denjenigen Passagen ab, in denen bloß der eigene gegen den Fetisch des anderen ins Feld geführt wird, hin zur Metaphorik des Diskurses, findet sich eine den gesamten Diskurs tragende fetischistische Konstellation: Eine intrikate Zweideutigkeit unterläuft den Vertretern des Diskurses in der dort fest verankerten Rhe-

»Übrigens, die Modevorschrift für den Klettersommer '88 lautet: Zum gelbroten Dolomitenfels trägt man das im komplementären Türkisgrün gehaltene Sweatshirt mit der hautengen hellblauen Clanzhose mit zarten, die Beinform betonenden, vajoletroten Längsstreifen, dazu ein dezentes graues Frotteestirnband mit schmalen Rändern im Hellblau der Hose sowie einen farbengleichen chalk-bag. Zum grauen Granit der Urner Alpen dagegen [...] «- und so fort. Hier wird Ende der Achtziger humoristisch die Infiltration des Haindlkars (direkt bei Schneiders Route in Über Raben gelegen) durch »Modefritzen« beklagt (aber auch durch Vertreter der urbanen Intellektuellenschicht: "Was können sie denn schon? Den achten Grad klettern und über Marcuse diskutieren.« - Putz, Mit Bauz im Haindlkar. In: ZDÖAV 1988, S. 37f., hier S. 37). 1992 stellt Peter Baumgartner im Alpenvereinsjahrbuch mit kaum verhohlener Besorgnis fest, dass Bekleidungsmode im Lauf der 70er und 8oer Jahre tatsächlich auch in den Bergen angekommen ist (vgl. Baumgartner, Die wahren Abenteuer sind im Kopf. In: ZDÖAV 1992, S. 49-52, hier S. 49). Im selben Jahrbuch konstatiert Henner Schülein, die »]agd auf die Waren «stehe quer zur (hier normativen) »Lebenshaltung des Bergsteigers«, diese sei »asketisch und nicht hedonistisch « (Schülein, Die Indianer Europas. In: ZDÖAV 1992, S. 53-60, hier S. 60). Hinter farblicher Cestaltung (aber auch so mancher technischen Weiterentwicklung) vermutet der Autor »das Prinzip der Absatzsteigerung durch Produktdifferenzierung", was dem eingeforderten asketischen Bergsteigerethos natürlich krass entgegensteht (S. 55). Schülein, Die Indianer Europas. In: ZDÖAV 1992, S. 53-60, hier S. 56. 
torik der Anthropomorphisierung des Berges. Zum Grundstock alpinistischer Verständigung gehören, der Kulturwissenschaftlerin Dagmar Günther zufolge, erotische Metaphern; ${ }^{14}$ dasselbe kann man schon in Ulrich Aufmuths wirkmächtiger Psychologie des Bergsteigens von 1984 nachlesen. Zahlreiche Fundstellen belegen hier die sexualisierte Bergmetaphorik: »Unser Berg entschleierte sich zuweilen für einen Augenblick, als ob er eine gefallsüchtige Schöne wäre, und sah oben reizend, unten aber sehr geheimnisvoll aus «, wird etwa Edward Whymper zitiert, bei Leo Maduschka liest man vom Gestein, das »umworben und geliebt werden kann wie eine Frau ${ }^{15}$; Aufmuth selbst führt diese Reihe im Versuch einer psychologischen Rekonstruktion fort: »Welches Lustgefühl geht durch uns hindurch, wenn die Steigeisen im harten Firn so richtig eindringen und knirschend greifen! ${ }^{16} \mathrm{Zu}$ gleich aber ist im alpinen Sprachgebrauch mit der Metaphorik des Krieges bzw. des Kampfes auch ein aggressives Moment tief verwurzelt. In den von Aufmuth angeführten Belegen ist die Rede vom >Kampf ebenso oft anzutreffen wie die vom Berg als Feind, als >furchtbarem Gegner $<{ }^{17}$ militärischer Herkunft sind etwa die Bezeichnungen >Belagerung oder auch >Biwak` (das ursprünglich einen kampierenden Wachposten auf freiem Feld meint, die >Beiwacht`). ${ }^{18}$

Der Berg ist sowohl die zu gewinnende Frau als auch der zu besiegende Feind, Erotik tritt neben Aggression. Die Rhetorik des Diskurses zeugt dementsprechend von einer höchst ambivalenten Belebung unbelebter Gegenstände (hier: des Felsens) - die Archive von Alpinismus und Fetischismus lassen sich an markanten Punkten in Deckung bringen. In einer Formulierung Eugen Guido Lammers, eines der bekanntesten Alpinisten in der ersten Hälfte des 20. Jahrhunderts, findet dieses Verhältnis zum Berg seinen zum Paradox zugespitzten Ausdruck: »Wohl lieben auch wir die stolzen Berge leidenschaftlich und treu, doch nicht träge genießend, sondern wir lieben sie hassend und bekämpfend, wir lieben sie erst, wenn sie besiegt unter unserem Fuße liegen ${ }^{19}$.

14 Vgl. Günther, Alpine Quergänge (1998), S. 206-211.

15 Edward Whymper und Leo Maduschka zit.n. Aufmuth, Zur Psychologie des Bergsteigens (1988 [1984]), S. 151.

16 Ebd., S. 152f. Die Passage als ganze lautet: »Welche Wonne ist es, die Hand auf kompakten, sonnenwarmen Fels zu legen, sich anzuschmiegen an eine pralle Wand, einen festen, handfüllenden Criff zu packen! Welches Lustgefühl geht durch uns hindurch, wenn die Steigeisen im harten Firn so richtig eindringen und knirschend greifen! Welch gutes Cefühl, wenn wir den Pickel in eine solide Schneedecke hineinstoßen. «Aufmuth hält aber doch auch fest: »Ein Berg und eine Frau sind auf der materiellen Ebene zweifellos etwas sehr Verschiedenartiges« (S. 152).

17 Vgl. ebd., S. 166-173.

18 Anfang der 1990er findet der Sachverhalt im Alpenvereinsjahrbuch auch seinen sprachwissenschaftlichen Niederschlag: Kirch, Wie die Bergsteiger reden. In: ZDÖAV 1989, S. 117-124, hier S. 118. 


\section{Die naheliegenden Fetischismen auch in Über Raben}

Viele dieser im kulturellen, hier: alpinistischen Kontext bereitliegenden fetischverdächtigen Topoi finden sich auch in Über Raben. Der Berg wird auf verschiedenste Weise mit Sexualität in Zusammenhang gebracht: Dem Blick einer Kollegin meint Schneider beispielsweise den Verdacht ablesen zu können, dass der Berg auch einen Ersatz dafür darstellt (ÜR 61). ${ }^{20}$ Dass dieses metaphorische Verhältnis auf Figurenebene durchaus reflektiert wird, ist den an Arno Schmidts Karl-May-Lektüre geschulten Blödeleien unter Kletterern abzulesen, an die sich Schneider erinnert:

Dann hatten sie sich Arno Schmidts Deutungen der Landschaften bei Karl May hingegeben und den Spekulationen darüber, wie er sich wohl dem Bergsteigen genähert hätte. Tagelang waren sie an Sätzen hängen geblieben wie: `Er legte behutsam einen Klemmkeil der Größe drei in die Spalte.< Oder: >Die Höhlung ist gerade so weit, dass die Kuppen von Zeige- und Mittelfinger bequem hineinpassen. (ÜR 115)

Dieser Schmidt-Extrapolation geht in der Erinnerung ein Dialog zur technischen Bestückung des Berges voraus, genauer: zur »fanatischen Haltung gewisser Leute, unter keinen Umständen ein Stück Eisen« - etwa einen Sicherungshaken - »in die heiligen Berge zu treiben. [...] >Deflorationsphobie`, hatte Robert Fauler immer wieder gesagt, >die Angst des Kletterers vor der blutigen Realität der Penetration« (ÜR 115).

Auch anderweitig wird im Text die Nähe von Sexualität, Gewaltsamkeit und materiellem Artefakt nahegelegt; nicht explizit in der Wahrnehmung des Protagonisten zwar, aber aus den Details bzw. der Abfolge dieser Details ableitbar. Als er am zweiten Tag in der Haindlkar-Hütte erwacht, bilden seine ersten Wahrnehmungen ein verräterisches Arrangement, das eine hoch effiziente Masturbationsfantasie befeuert. So wird das Vorhandensein des Gewehrs registriert - »Das Weatherby hing an seinem Platz« (ÜR 52) - , unmittelbar darauf das einer morgendlichen Erektion. Nach einem Gedankengang, der sich möglichen Beischläfern seiner Exfrau widmet, führt die Gemengelage aus Psyche, Physis und Artefakt zur logischen und im Blick des Antifetischisten verräterischen - Abreaktion: »Er leuchtete noch einmal auf das Gewehr. Dann öffnete er die Tür und trat ins Freie, nackt wie er war. Die Sache mit dem Abwichsen hatte sich innerhalb von zwanzig Sekunden erledigt.«(ÜR 53)

20 Diese These, der zufolge Alpinismus als Ersatz für Ceschlechtsverkehr dient (und die im Roman von Schneider explizit zurückgewiesen, um nicht zu sagen: abgewehrt wird), findet sich im Detail auch bei Aufmuth, vgl. Aufmuth, Zur Psychologie des Bergsteigens (1988 [1984]), S. $151 \mathrm{ff}$. 
Auch des Warenfetischismus macht sich der Protagonist verdächtig: Während in alpinen Verhältnissen die Zweckrationalität der Mittel ästhetische oder andere Aspekte eigentlich ausschließen sollte, befördert im Gegenzug gerade diese Umgebung seine irrationale Bindung an Ausrüstungsgegenstände:

Er dachte an seine LCD-Stirnlampe von Petzl, seinen Millet-Fleecepullover, an seine beiden violett eloxierten Schraubkarabiner von Stubai. Es war kindisch, aber zu Ausrüstungsgegenständen konnte er eine Beziehung entwickeln, die zumindest so innig war wie zu Menschen. »Wie ein alter Mann«, hatte Elvira manchmal gespottet, und er hatte darauf gesagt: »Dann bin ich von klein auf ein alter Mann gewesen. «In Wahrheit hatte sie selbst ihre dottergelbe Northface-Jacke und ihren grünen Steinschlaghelm, der so abgewetzt war, dass keiner mehr die Marke erkennen konnte, geliebt wie Schoßtiere, aber das durfte man ihr nicht sagen. (ÜR 56f.)

Fast klingt das wie eine Paraphrase der oben angeführten Passage aus dem alpinistischen Archiv, in der dem Fetisch des Althergebrachten gefrönt wird (abgesehen von der Nennung der Markennamen). Zur tiefen Bindung zu langerprobten, sichtlich gealterten Artefakten liefert Über Raben auch das Gegenstück: die komplementäre Vorliebe für neue, modische Accessoires: Am Parkplatz beim Anbringen der Steigfelle auf den Schiern bemerkt der Protagonist: »Die Felle waren grellorange; eigentlich absurd, doch seit es diese bunten Felle gab, hatte er die altmodischen schwarzen unerträglich gefunden« (ÜR 10). Dass der Protagonist hier dem nach Haug - Missverhältnis von >Warenhaut und Warenkörper e erliegt, bleibt ihm selbst unerklärlich. Zugleich mit den Bemühungen der Industrie um ästhetische Alleinstellungsmerkmale wird so Schneiders eingestandene Wehrlosigkeit gegenüber solchen Schachzügen herausgestellt.

Indem die Gesäuse-Kapitel von Über Raben also eine Anzahl skandalöser Dingbeziehungen aufweisen, lässt sich die Perspektive Schneiders mit dem alpinistischen sowie dem Fetischdiskurs gleich mehrfach zur Deckung bringen. Zugleich sind aber die angeführten Fetischismen ausgesprochen naheliegend. Idiosynkratische Vorlieben für tote Dinge, die Anthropomorphisierung der Natur, die warenästhetische Kontaminierung von Zweckrationalität, das Artefakt als (buchstäblich) potenzsteigerndes Mittel, allesamt sind diese Verhältnisse so grundfetischistisch wie weitverbreitet und damit nicht besonders interessant. Man könnte sogar einwenden: Was sonst als fetischistische Gedanken sollen durch jemandes Kopf gehen, der allein und ausschließlich von Dingen umgeben ist? Der Fetischismus wäre dann bloß eine Folge eines suggestiven >Settings ${ }^{21}$ 
Mit der Feststellung der Kongruenzen zwischen den Diskursen ist über den Text kaum mehr gesagt, als dass er eben die Überschneidung der Diskurse wiedergibt, wie sie vom Archiv und, auf Ebene der Handlung, vom Setting nahegelegt bzw. vorgeführt werden. Worauf läuft ein solches Einführen fetischistischer Verhältnisse in die Berge hinaus? Eine mögliche Antwort würde die gemeinhin angenommene lebensweltliche Distanz zwischen Alltag und alpinem Abenteuer in Anschlag bringen, um daraufhin zu argumentieren, dass die Konstellation im Text zeigt, wie wenig stabil, wie porös diese Unterscheidung ist; eine imaginäre Linie zwischen Alltag und Abenteuer, wie sie auch zwischen Arbeit und Freizeit, Gesellschaft und Natur, Kultur und Körper, Marktgesetz und Naturgesetz, Warenästhetik und Gebrauchswert, Verblendungszusammenhang und Autonomie nicht undurchlässig bleibt. Der Kollaps dieser Unterscheidungen würde anhand der Tatsache demonstriert, wie alltägliche Fetischismen das Subjekt auch in der alpinen Extremsituation noch determinieren. Dieser Lösungsansatz würde zweifellos ein Licht auf die hier festgestellte Überschneidung von Alpinismus- und Fetischismusdiskurs werfen.

Über Raben jedoch stellt verdächtige Mensch-Ding-Verhältnisse auch an unerwarteter Stelle zur Diskussion: innerhalb des Zweckrationalen. Fetischismus und Fetischhandlungen sind unabhängig von der jeweiligen Diagnose zumeist mit dem Stigma des Irrationalen versehen. Das Rationale ist das (Lebens-)Notwendige, das Irrationale aber (Aberglaube, Perversion, Conspicuous Consumption, Kunst, Kultur ...) muss man sich erst einmal leisten können. Nun wird das Gebirge landläufig als lebensfeindliche Umgebung beschrieben; es sei kein Ort der Aus- oder Abschweifung, sondern ein Ort der Notwendigkeit. Überleben sei in seinen topographischen und klimatischen Verhältnissen nur möglich, wenn man sich (und sein Verhältnis zu den Dingen) dem Diktat des Zwecks mit annähernder Ausschließlichkeit unterwirft. Umso interessanter ist die Frage nach möglichen Residuen eines wie auch immer gearteten Fetischismus gerade in diesem Reich des Unabdingbaren, im Hantieren mit dem Lebensnotwendigen.

Dort, ganz grundlegend mit der Tätigkeit des Bergsteigers verbunden, liegen womöglich Verhältnisse vor, die viel profunder Fetischismus und alpinistischen Ding-Gebrauch kurzschließen. Ein unauffälliges und umso gravierenderes Überschreiten der geheiligten Grenze zwischen Mensch und Ding zeigt sich womöglich auch im rein sachlichen Hantieren mit Werkzeugen (Schiern, Haken, Gewehren) das dann so >rein n nicht mehr wäre. Die Fetischismen an der Oberfläche des Textes wären dann den tief in den dargestellten Tätigkeiten verankerten Fetischismen vorgelagert, würden den Blick von diesen ablenken, aber zugleich den Ort markieren, an dem sich eine Suche lohnt.

zimmer, das zur Anregung der Übertragung mit archäologischen Objekten ausgestattet war. Vgl. Böhme, Fetischismus und Kultur (2012 [2006]), S. 387f. 
Deswegen wird im Folgenden das Verhältnis des Protagonisten zu seinen technischen Artefakten vor einen mehrfachen Hintergrund gestellt: vor das Archiv des Alpinismus und die Folie des Fetischdiskurses zum einen, zum anderen aber auch vor technikphilosophische Kontexte. Dem Verhältnis des Protagonisten zu seinen technischen Hilfsmitteln sind verschiedene Haltungen ablesbar - die Erweiterung des Menschen durch Technik, aber auch seine Unterwerfung, seine Verschmelzung mit dem Artefakt. Diese Haltungen werden nun auf ihre technikphilosophische $\mathrm{Zu}$ gehörigkeit hin untersucht, dann die Konsequenzen für den Text herausgestellt.

\section{Alpinismus und Technik}

\section{Unangemessene Vorüberlegung}

Schneiders Aufenthalt im Gesäuse ist durchgehend bestimmt und ermöglicht durch den Einsatz technischer Mittel. Umfangreiche Packlisten zeugen davon: Die Ausübung eines Sports, besonders eines solchen in lebensfeindlicher Umgebung, bedarf einer Unzahl an Dingen. Alpinismus ohne Technik ist, zumal im Winter, nicht vorstellbar; dennoch ist ihr Einsatz im gesamten alpinistischen Diskurs umstritten.

Das liegt daran, dass Technikeinsatz von einer vorgängigen Notwendigkeit ausgeht. Gerade das Wort >Notwendigkeit`aber hat im Zusammenhang des Alpinismus einen zweifelhaften Klang: Vordergründig >muss< kein Mensch bergsteigen (abgesehen von denen, die am Berg ihr Geld verdienen). Es ist geradezu Kernstück alpinistischer Selbstverständigung (und, wie erwähnt, hochgradig fetischverdächtig), dass auf der Zweckfreiheit des Unterfangens bestanden wird; siehe etwa Lionel Terrays vielzitierte Formulierung vom Alpinismus als der >Eroberung des Nutzlosen ${ }^{22}$. Durch das Betreten der Alpen wird eine gewissermaßen zwecklose Notwendigkeit überhaupt erst geschaffen. Die Berge und die zahlreichen Möglichkeiten, dort sein Leben zu verlieren, stellen eine Notlage bereit, der dann beizukommen Inhalt der Kulturtechnik >Alpinismus ist. So steht jeder vermeintlich rationale Materialeinsatz - und das ist sicher mit ein Grund für die anhaltenden Diskussionen zum Thema - im Alpinismus unter zur Gänze irrationalen Vorzeichen. Auf zweckrationalem Boden lässt sich hier nicht argumentieren: Das zweckdienlichste Werkzeug ist immer noch so verrückt wie eben dieser Zweck.

Es verwundert nicht, dass sich bei einer auf dieser Grundlage geführten Diskussion zum Technikeinsatz in den Argumentationsmustern, Werthaltungen und

22 Vgl. Terray, Vor den Toren des Himmels (1965). Vgl. auch Aufmuth, Zur Psychologie des Bergsteigens (1988 [1984]), S. 11. 
Abgrenzungsmanövern des alpinistischen Diskurses großflächige Überschneidungen mit dem Fetischdiskurs finden. Schon vor dem Blick ins Archiv, schon bei der logischen (und damit dem Thema sicherlich höchst unangemessenen) Betrachtung dieses seltsamen Verhältnisses von künstlicher Notwendigkeit und ihrer technischen Bewältigung drängen sich einige dem Fetischismus analoge Aporien auf. Vor dem Eintritt ins Archiv wird nun also zur Klärung der Grundlagen eine, sagen wir, befremdete Außenperspektive eingenommen.

Die grundlegende Aporie des alpinistischen Werkzeugs ist aus einer solchen Perspektive, dass die vollständige Erfüllung seines Zwecks diesen selbst obsolet machen würde: Seine absolute Zweckmäßigkeit müsste - fatalerweise! - die erwünschte Strapaze, das aufgesuchte Risiko restlos ausschalten. Alpinismus ist das Verlassen der Zivilisation, des (nach Arnold Gehlen) ১technischen Nests`, jedoch zwangsläufig mit technischen Mitteln. Gerade durch die Dinge, die ihn überhaupt erst ermöglichen, wird so der Alpinismus in seiner Existenz gefährdet. Das zeigt sich nicht nur in jedem einzelnen Fall von Werkzeuggebrauch, in dem das Werkzeug das Verdienst des Bergsteigers umso mehr schmälert, je besser es für seinen Zweck geeignet ist, das zeigt sich auch in der historischen Entwicklung des Alpinismus, wie sie im Diskurs regelmäßig rekapituliert und aktualisiert wird: Körperliche Ausgesetztheit, existenzielle Bedrohung steht im Verlauf der technischen Entwicklung potenziell immer kurz davor, von den technischen Mitteln eingeholt $\mathrm{zu}$ werden. Jedes Vorhaben muss nach seiner technischen Bewältigung unverzüglich erschwert werden (damit man weiter bergsteigen kann), sodass der Einsatz neuer Technik notwendig wird, woraufhin sich das Spiel wiederholt. ${ }^{23}$

Das Verhältnis von freiwillig herbeigeführtem, zugleich aber existenziellem Risiko und Strapaze zu ihrer zwangsläufig und notwendig niemals vollständigen materialen Bewältigung kann man als dialektische Spannung bezeichnen - aber auch als eine ganz grundlegend fetischistische Konstellation. Die den Alpinismus ausmachende Mischung aus Angst, materialem Behelfsmittel und nicht zuletzt Wiederholungszwang nämlich findet sich auch im Fetischdiskurs. Die Parallele zum Fetischismus besteht hier nicht in den Ursachen (dem frühkindlichen Trauma), sondern im Ablauf von Herausforderung und Ding-Einsatz: Eine Situation wird

23 Den Widerstreit, der in einer solchen Tätigkeit liegt, die einerseits ideologisch technikkritisch, betont irrational, andererseits aber technisch ermöglicht und vermehrt einer technischen Ratio unterworfen ist, fasst Kaufmann unter Berücksichtigung eines repräsentativen alpinistischen Textkorpus zusammen: »Es mag sein, dass Bergsteigen - vor allem extremes Bergsteigen - seinen Sinn nicht zuletzt daraus bezieht, den Regeln und Normen einer durchorganisierten industrialisierten Welt zu entfliehen. [...] Zugleich aber untergräbt der Einzug technischer Systeme und daran angekoppelter Dienstleistungen zentrale Momente der Gegenwelt des Bergsteigens. Nicht zuletzt die Eigenverantwortlichkeit, die Selbstbestimmung und -beherrschung des Risikos werden tendenziell eskamotiert.« - Kaufmann, Technik am Berg (2006), S. 111. 
hergestellt, immer und immer wieder, deren existenzieller Bedrohlichkeit nur mit Hilfe des mitgebrachten Dings beigekommen werden kann. Überspitzt formuliert: Der Bergsteiger sucht den Berg auf wie der Fetischist den Akt; die eigentliche Begegnung aber mit dem auf Wiederholung insistierenden Realen wird durch das dazwischengeschobene Artefakt stets unterbunden (plaisir statt jouissance!). Nicht nur das Lustprinzip, auch das Prinzip der Funktionsunterwäsche ist das der Homöostase. ${ }^{24}$

Die implizite Ratio der Packliste - und die alpinistische Fachliteratur ist voll von solchen Listen, immerhin ist Alpinismus nicht zuletzt Werkzeugkunde - ist Vollständigkeit, und mit der Vollständigkeit der Ausrüstung ist es dasselbe wie mit ihrer Zweckmäßigkeit: Ihr eigentlicher Zweck wäre obsolet, wäre sie jemals erreicht, was aber ohnehin logisch unmöglich ist. Wer alles mitnehmen könnte, bräuchte gar nicht erst aufzubrechen. Vollständigkeit als nicht einzuholendes Ideal ist dennoch das Phantasma, an dem sich der prototypische Alpinist beim Packen seiner Sachen zwangsläufig orientiert und das von den Ausrüstungslisten in den Erfahrungsberichten, der Ratgeberliteratur und nicht zuletzt in den Katalogen ausgiebig bedient wird. ${ }^{25}$ Im großzügig bemessenen Netz, das der Fetischdiskurs auswirft, verfängt auch diese Gemengelage aus existenzieller Bedrohung und imaginärer Vollständigkeit: In der exponierten Position des Alpinisten, der nie sendgültig، ausgerüstet sein wird, findet sich dabei der grundlegende Mangel gespiegelt, der in Lacan'schen psychoanalytischen sowie in an Lacan orientierten warenästhetischen Fetischtheorien im Kern des Subjekts liegt; im implizierten Telos imaginärer Vollständigkeit spiegelt sich die fetischistische Verleugnung der Kastrationsdrohung. Der Bergsteiger überführt, so ließe sich diese Analogie fortführen, den existenziellen Mangel in einen konkreten, er sucht in fetischistischer Ambivalenz die Situation des Mangels auf, gegen die er sich gleichzeitig nach Kräften wappnet.

\section{Technikdiskussion im Alpinismus}

Alpinismus in seinen technischen Belangen ist also, wie die eben erfolgte Zuspitzung illustriert, eine zutiefst paradoxe Angelegenheit, und für Anklänge an diverse Fetischdiskurse braucht man nicht lange zu suchen. Tatsächlich wirken die eben

24 »[D]as Lustprinzip ist Prinzip der Homöostase.« - Lacan, Unbewusstes und Wiederholung (1978 [geh. 1964]), S. 37.

$25 \mathrm{Zu}$ jedem Ratgeber gehören Ausrüstungslisten, zu jeder Tourenbeschreibung die Angabe notwendiger Hilfsmittel. Kaufmann spricht von ca. 50 Gegenständen, die in gegenwärtigen Lehrbüchern allein unter dem Aspekt `Sicherheit sabgehandelt werden; vgl. Kaufmann, Technik am Berg (2006), S. 110. An die 70 verschiedene Ausrüstungsgegenstände gibt der Ratgeber Alpine Ausrüstung von 1984 für Fels- und Eistouren ab mittlerer Schwierigkeit an (vgl. Hanschke, Alpine Ausrüstung (1984)). 
eher spekulativ festgestellten Aporien auch ganz konkret innerhalb des alpinistischen Diskurses, speziell in der nie abbrechenden Diskussion um den Einsatz technischer Hilfsmittel.

Die Ratio, nach der in diesen Technikdiskussionen Urteile gefällt werden, kann entweder die höhere Sicherheit, die größere Erfolgsaussicht und die Verschiebung der Leistungsgrenzen oder aber die Bewahrung des alpinen Charakters bzw. des Charakters des Alpinisten sein. Die einen sehen den Alpinismus stets durch Technik bedroht, die anderen in einer neuen, nichtsdestoweniger seigentlicheren überhaupt erst ermöglicht. ${ }^{26}$ Regelmäßig wurde angesichts technischer Neuerungen von der einen Seite das Ende, von der anderen Seite erst der Beginn des >wahren Alpinismus verkündet. ${ }^{27}$

Seine sicher deutlichste Ausprägung erhält der Technik-Konflikt im Alpinismus, ${ }^{28}$ wenn es um die Sicherung und Fortbewegung mit Hilfe von Haken geht. Die ersten Haken waren bloße Eisenstifte, oft einfache Mauerhaken. Die Einführung des sogenannten Fiechtlhakens 1910, einer aus einem Stück geschmiedeten Kombination aus Stahlstift und Öse, erleichterte das Befestigen von Seilen am Fels und ermöglichte den Einsatz von Seilzügen und Pendelquergängen; ein solchermaßen technisch gestütztes Klettern führte zum vehementen Einspruch von Kletterpuristen wie Paul Preuß, der 1911 darauf drängte, sich doch auf Routen zu beschränken, die man gänzlich ohne Technikeinsatz gehen konnte; ${ }^{29}$ eine Einstellung, die viele Anhänger fand, sich aber nicht durchsetzte; stattdessen dominierten noch jahrzehntelang technische Begehungen im Stil Hans Dülfers, trotz mahnender Erinnerung an die karge Ausrüstung der Pioniere und trotz des Verächtlichmachens der >Nagelfritzen als »Sklaven des Werkzeugs «. ${ }^{30} 1936$ veröffentlicht Karl Ziak den

26 So wurde etwa Lammer, der 1937 vehement gegen den Gebrauch des Hakens wettert, 50 Jahre davor vorgeworfen, die damals neue, von ihm vertretene Schule des führerlosen Alleingangs sei eine »verwerfliche und den Alpinismus schädigende«Praxis. - K. Schulz zit.n. Pichl, Wiens Bergsteigertum (1927), S. 35.

27 Wie auch Elmar Landes feststellt: »Nie hatte es mit dem Bergsteigen noch was zu tun, wann immer eine junge Generation von Bergsteigern sich andere Wege zum Ziel erkor als die alte. Oder die Wege zum Ziel in anderer Manier zu gehen sich anschickte. Oder Wege zu anderen Zielen suchte. Umgekehrt sahen sich die Bergsteiger in der Gefolgschaft des Trends zum Neuen selten imstande, das, was >die Alten < trieben - und wie sie's trieben, noch als Bergsteigen gelten zu lassen.«- Landes, Bergsteigen oder nicht? In: ZDÖAV 1991, S. 55-61, hier S. 55; vgl. schon Jennewein, Vom Prinzip der Auslese. In: ZDÖAV 1954, S. 5-12, hier S. $7 f$.

28 Die historischen Eckdaten entnehme ich den folgenden Texten: Schubert, Bohrhaken im Widerstreit der Meinungen. In: ZDÖAV 1986, S. 229-243; Kaufmann, Technik am Berg (2006); Grupp, Faszination Berg (2008).

29 Vgl. Preuß, Künstliche Hilfsmittel auf Hochtouren (1911). Zu den Erwiderungen siehe etwa Nieberl, Künstliche Hilfsmittel auf Hochtouren (1911).

30 Oskar Erich Meyer zitiert nach Schmitt, Vom Gipfelkult bis zum 6. Grad. In: ZDÖAV 1962, S. 2948, hier S. 4of. 
schon zitierten Band Der Mensch und die Berge, eine konservative Abrechnung nicht nur mit alpinistischem Sportsgeist, der die Berge zum »Turngerät«, zur »Zirkusarena« mache, sondern auch mit dem Einsatz technischer Mittel, der schwierige Routen zu »Spaziergängen « degradiere (eine Ansicht, die zu Preuß' Zeiten noch als >sportlich galt). ${ }^{31}$ Im Folgejahr unterschied Eugen Guido Lammer, wie Ziak ehemaliger Alpinpionier und eine gewichtige Stimme im Diskurs, in Wie anders ist das Besteigen der Alpen geworden! streng zwischen solchen Hilfsmitteln, die die Fortbewegung >natürlicher<, spurloser machen (wie Steigeisen, Schi, aber auch das besonders leichte Zdarsky-Zelt), und solchen, die sich an der natürlichen Unberührtheit der Berge vergehen und damit auch die Seele des Alpinismus austreiben würden: neben versicherten Steigen, Hütten über der Ödlandgrenze und der Prusikschlinge zum Aufstieg am Seil prominent eben der Fiechtlhaken, der den Bergsteiger "unentrinnbar versklavt und sein Tun würdelos macht « ${ }^{32}$. Unmittelbarer Kontakt zum Berg bis hin zur Selbstauflösung war das bergsteigerische Ideal (das stets von Bekenntnissen zu diesem oder jenem dann doch als snatürlich eingestuften Hilfsmittel unterlaufen wurde).

Durch zwei Weltkriege beschleunigte sich die technische Entwicklung des Alpinismus; der 1944 erfundene Bohrhaken - der für seine Anbringung nun nicht mehr auf Felsspalten und -ritzen angewiesen war - setzte sich wie der Fiechtlhaken trotz prominenter Gegner (etwa Walter Bonatti) durch. Bis zum Ende der sechziger Jahre war mit seiner Hilfe, so entstand der Eindruck, jede Schwierigkeit im Gebirge besiegt. In sogenannten Bohrhakendirettissimas wurden glatte Wände in Fallinie durchstiegen; unter enormem Materialaufwand bewegte man sich mit Trittleitern und Fixseilen vorwärts. In den Alpenvereinsjahrbüchern dieser Zeit wird wenig überraschend den technischen Kletterern (auch schon der Zwischenkriegszeit) bescheinigt, »trotzdem echte Bergsteiger [...] und ganze Männer « ${ }^{33} \mathrm{zu}$ sein. Kletter-Schwierigkeit nicht nur an der Felsbeschaffenheit, sondern an der Anzahl der Sicherungshaken zu bemessen, ist nun selbstverständlich, und dass es seit den zoern neben der technischen auch eine sportliche Weiterentwicklung gegeben haben könnte, wird von einem Autor stark bezweifelt. ${ }^{34}$ An derselben Stelle etwas später stößt man dafür auf die Rede vom >Epigonentum ‘ der >Eisernen Zeit<, der fortschreitenden >Erschlosserung ‘ der Alpen; so detailliert wie im Ton despektierlich werden die großen Materialschlachten der technischen Direttissimas aufgezählt. ${ }^{35}$ Bald darauf fiel Messners im gesamten weiteren Diskurs vielzitiertes Diktum vom mittels Bohrhaken und Co. begangenen »Mord am Unmöglichen«;

Vgl. Ziak, Der Mensch und die Berge (1936), S. 183, 186.

32 Lammer, Wie anders ist das Besteigen der Alpen geworden! (1937), S. 29.

33 Maix, Menschen im Haindlkar. In: ZDÖAV 1956, S. 111-119, hier S. 114.

34 Vgl. Hausmann, Die modernen Felsfahrten im Wetterstein. In: ZDÖAV 1954, S. 93-103.

35 Vgl. Schmitt, Vom Gipfelkult bis zum 6. Grad. In: ZDÖAV 1962, S. 29-48, hier S. 40-43. 
über die psychologischen Folgen schreibt er: »Der Mut wird in Form von Haken im Rucksack mitgetragen. ${ }^{36}$

Ab den 1970ern kam es zu einer Rückbesinnung auf die Prinzipien Paul Preuß', vor allem durch den Einfluss des amerikanischen Freeclimbing. Haken wurden zwar nicht grundlegend abgelehnt, aber meist nur zur Sicherung verwendet. Größtenteils fand hier die fortschrittliche und verlässliche Bohrhakentechnik Anklang, traditionelle Alpinisten jedoch bevorzugten nun mitunter den hergebrachten Haken und andere, schwieriger zu setzende Sicherungsmittel - vor allem in Hinsicht auf die psychologische Schwierigkeit, die durch verlässliche Sicherungen zu stark vermindert würde. Im Rahmen der sogenannten >Bohrhakenkriege wurden Standund Sicherungshaken mit Gewalt entfernt und teilweise angesägt, mitunter mit Todesfolge. Diskutiert wurde über die Heranzüchtung von >Nichtskönnern`, die Erziehung zur Unselbständigkeit, das Klettern im >Einheitsmaß<, die Vergewaltigung der Berge und so fort. ${ }^{37}$ Anfang der $90 e r$ Jahre legten sich die Auseinandersetzungen langsam. ${ }^{38}$

Regelmäßig wurde bei all dem die Geschichte der technischen Entwicklung und der sie begleitenden Diskussionen rekapituliert; wenn ein Autor 1991 schreibt: »Mit nur geringfügig variierenden Inszenierungsideen erfuhr diese Version des Dramas verschiedene Wiederholungen ${ }^{39}$, dann gilt dasselbe für die sich wiederholenden Resümees dieses Dramas. Mehrfach stößt man ab Ende der 1980er auch auf die Feststellung, die Diskussion würde nun nicht mehr geführt - weil, so der Subtext und manchmal der Text, mittlerweile ohnehin ausgemacht sei, welcher Standpunkt der richtige sei; oder weil sich allgemeine Toleranz breitgemacht habe (wobei erneut klar sei, wer hier eigentlich richtig liege ...) usf. In direkter Nachbarschaft zu solchen Texten findet sich dann der eine oder andere, der exakt diese Diskussion eben doch weiterführt und dabei keineswegs zur Toleranz neigt. ${ }^{40}$

36 Messner, Direttissima - oder Mord am Unmöglichen (2002 [1968]), S. 73.

37 Vgl. zur Diskussion zusammenfassend Schubert, Bohrhaken im Widerstreit der Meinungen. In: ZDÖAV 1986, S. 229-243; ders., Mehr Sicherheit am Berg durch einwandfreie Standhaken. In: ZDÖAV 1973, S. 194-198, hier S. 194; ders., Der Hakenkrieg im Wilden Kaiser (2000); Dick, Können Haken Sünde sein? In: ZDÖAV 1997, S. 137-149.

38 Wie es einem alle Standpunkte relativierenden Frieden wohl entspricht, findet sich im Jahrbuch von 1988 der liebevoll-ironische Rückblick in Form der Darstellung eines Alpinisten, der >immer noch<Fiechtlhaken verwendet; das ritualhafte Einschlagen des Hakens wird dabei im Text, seinen unironischen Gegenstücken ganz entsprechend, mit religiösen ebenso wie mit erotischen Assoziationen versehen. Vgl. Putz, Mit Bauz im Haindlkar. In: ZDÖAV 1988, S. 37f., hier S. 38.

39 Landes, Bergsteigen oder nicht. In: ZDÖAV 1991, S. 55-61, hier S. 55.

401992 verkündet Baumgartner: »Die Diskussion um die Entartung oder Reinerhaltung des Alpinismus wird nicht mehr geführt«, während Schülein im selben Heft angesichts der von oben eingebohrten Sportkletterrouten vor der »Herrschaft des Technikers« im Berg warnt; 1995 meint Roeper, die Diskussion wäre vorbei, weil »im Zweifelsfalle« klar sei, welcher Stil 


\section{Die Diskussion in zeitlicher und räumlicher Nähe zur Rosskuppen-Dachl-Verschneidung}

Die Rosskuppen-Dachl-Verschneidung - in der Schneider in Über Raben auf seine Verfolger wartet - wurde 1936 von Fritz Sikorovsky und Raimund Schinko erstmals durchstiegen, unter Zuhilfenahme damals umstrittener technischer Hilfsmittel. Gerade die schon zitierten tendenziell bis radikal konservativen Streitschriften von Ziak und Lammer, in denen der Einsatz technischer Neuerungen am Berg - mit wenigen Ausnahmen - verdammt wird, stammen nun exakt aus dieser Zeit. Konzentriert werden hier Topoi alpinistischer Technikkritik angeführt, die die Diskussion davor und noch lange danach geprägt haben bzw. prägen werden.

Beide führen den Vorwurf an, dass der Gebrauch technischer Hilfsmittel schlicht Betrug sei, an sich selbst wie am Berg: Haken, Pendelquergänge, Steigbügel, künstliche Trittflächen etc. seien »Mittel der Bergüberlistung«, die »F l u c h t i n di e L ü g e« lasse den Menschen »naturferner« werden und »seelisch verarmen«. Dazu kommt der Vorwurf, dass man durch ein technisch-sportliches Vorgehen die naturgegebene Ganzheit des Berges zerstöre. ${ }^{41}$ Umso schwerer wiegt dieses Verdikt von Zerstückelung und Unterwerfung, wenn man die stark religiöse Konnotation des Berges bzw. des Bergerlebnisses bedenkt. Was auch immer einen Alpinisten in die »heiligen Höhen « treibe, »alle suchen sie doch etwas Höheres, Übermenschliches, Göttliches in den Bergen. « ${ }^{42}$ Nicht nur werden bei Ziak in diesem Zusammenhang Heroismus, Romantik und Erotik des Bergsteigens in Religion zusammengeführt, Alpinismus insgesamt wird der Religion gleichgestellt, die Alpinisten nach bekanntem Muster in Rechtgläubige und Häretiker geteilt - diejenigen, die die Übermacht, die Ursprünglichkeit und Ganzheit des alpinen Charakters achten, und solche, die sich ihren Berg zurechtstückeln und ihm mit künstlichen Mitteln zu Leibe rücken.

der bessere sei; 1997 vertritt Dick einen pointiert bis kämpferisch argumentierten Standpunkt und spricht zugleich von allgemeiner Toleranz; 1998 meint Martin Kind, die Tatsachen haben die Diskussion ohnehin überholt, die er und im selben Jahrgang Hans Peter Eisendle dann dennoch weiterführen: Letzterem zufolge würde etwa auch der vermeintlich ssaubere، Stil im Gebirge durch das gut gesicherte Training im Klettergarten entwertet. - Baumgartner, Die wahren Abenteuer sind im Kopf. In: ZDÖAV 1992, S. 49-52, hier S. 51; Schülein, Die Indianer Europas. In: ZDÖAV 1992, S. 53-60, hier S. 58; Roeper, »Wo soll das alles enden? «n: ZDÖAV 1995, S. 65-73, hier S. 68; vgl. Dick, Können Haken Sünde sein? In: ZDÖAV 1997, S. 137149; Kind, Alpinismus ad absurdum? In: ZDÖAV 1998, S. 203-211, hier S. 210; Eisendle, Was wird aus dem Bergsteigen? In: ZDÖAV 1998, S. 215-219, hier S. 215.

Ziak, Der Mensch und die Berge (1936), S. 180, vgl. 177; Lammer, Wie anders ist das Besteigen der Alpen geworden! (1937), S. 41. 
Nicht nur in religiöser Hinsicht stellt das Gebirge in den Augen seiner Verteidiger einen Gegenpol zum >weltlichen Leben dar; auch außerhalb religiöser Konnotationen spielt es bei Ziak wie Lammer die Rolle einer Gegenwelt zum Alltag. Die Alpinisten wollen "aus Maschinen, $\mathrm{zu}$ denen uns eine mechanisierte Welt gemacht hat, wieder [zu] Menschen [...] werden ${ }^{43}$, so Ziak. Dementsprechend auch Lammer:

Schaut auf, die ihr drunten im Tiefendasein von der Maschine geknechtet werdet, dort oben hat euch Cott ein Reich der Freiheit aufgebaut, ein ungeheures Land der Zuflucht [...]. Drunten stöhnt ihr in der Fron der Zwecke, dort oben aber spottet alles der Zwecke. ${ }^{44}$

Dieser Charakter einer Gegenwelt werde nun, so Lammer, durch den Technikeinsatz untergraben. ${ }^{45}$ Der Alpinist, der einen Nagel in den Berg schlägt, sei »nichts weiter als ruhig berechnender Handwerker«, das Klettern am Drahtseil (am Klettersteig) sei »[m]aschinenmäßig«, "geistloser, seelenloser Trott, [...] der Mutterfels [!] ist Nebensache, gleichgültig. ${ }^{46}$ Indem der Mensch den Berg technisch erschließt, opfert er die Gegenwelt einem technischen Maßstab, der ihn und sein Tun (siehe die Rede vom »Sklaven des Werkzeugs«) unterwirft. Eine dem durchrationalisierten Alltag entgegengesetzte, ihrem Ideal nach natürliche, technik- und zweckfreie Sphäre wird vor diesem Hintergrund beschworen und verteidigt, ${ }^{47}$ bedient wird damit der schon davor und noch lange danach kursierende Topos von

43 Ebd.

44 Lammer, Wie anders ist das Besteigen der Alpen geworden! (1937), S. 77.

45 Der Topos hält sich (zumindest) bis zum Ende des Jahrhunderts im Alpenvereinsjahrbuch: Indem der Alpinismus in dialektischer Spannung zugleich vorindustrielle Verhaltensweisen fördere und technisches Denken reproduziere, biete er, so ein Text von 1992, »erhebliches sozialkritisches Potenzial«; jedoch wird beklagt, dass der Alpinist, anstatt das Potenzial der Berge für atavistische Rückbesinnung anzunehmen, dem saggressiven technischen Fortschritt` willfahre und damit die kompensatorische Wirkung des Alpinismus selbst untergrabe. Vgl. Schülein, Die Indianer Europas. In: ZDÖAV 1992, S. 53-60, hier S. 53 f. 1998 resümiert ein anderer den Zusammenhang zwischen Wirtschaftswunder, Konsumrausch und dem Aufschwung des Kletterns, um mit der Klage zu schließen, dass im Zeitalter von Professionalisierung und Spezialisierung auch im Alpinismus »der Fachidiot [...] kein Phantom mehr« sei. Vgl. Kind, Alpinismus ad absurdum? In: ZDÖAV 1998, S. 203-211, hier passim u. S. 211.

46 Lammer, Wie anders ist das Besteigen der Alpen geworden! (1937), S. 38, $49 \mathrm{f}$.

47 Vgl. dazu aus dem Jahrbuch Lenk, »Den wirklichen Gipfel werde ich nie erreichen. «In: ZDÖAV 1985, S. 103-114; Mann, Wagnis und Ehrfurcht. In: ZDÖAV 1964, S. 174-182. Des Weiteren vgl. Aufmuth, Zur Psychologie des Bergsteigens (1988 [1984]), S. 19, 38 u.ö., ebenso die Rede von der »säkularen Form der Askese« in Viktor Frankls Ausführungen zum Alpinismus: Frankl, Der Alpinismus und die Pathologie des Zeitgeistes. In: ZDÖAV 1988, S. 61-63, hier S. 62. 
der Versklavung und Unterwerfung des Menschen unter eine technische Rationalität, wie er dann prominent etwa in der Kritischen Theorie begegnet. ${ }^{48}$

Ein Einwurf zur Rhetorik Lammers ist angebracht: Für die diskursive Landschaft bezeichnend ist, dass Lammer hier und anderswo die eigene Argumentation unterläuft, wenn er die Tätigkeit des Körpers beim Bergsteigen - eigentlich Gegenpol zur >Welt der Zwecke - nach Maßgabe arbeitsteiliger Effizienz beschreibt; allen Sinnesorganen kommen ihre jeweiligen Aufgaben zu, der Körper wird in seiner Darstellung zergliedert eher wie ein Mechanismus denn ein Organismus. ${ }^{49}$ Das widerspricht dem sport- und technikkritischen Standpunkt und bringt ihn in Sichtweite etwa zu dem, was dann bei Horkheimer/Adorno der technoid-faschistische Blick auf den Körper als auseinandernehmbarer Mechanismus ist. ${ }^{50}$

Bei aller latenten Widersprüchlichkeit der Argumentation (die nicht in jeder Lektüre schlagend werden muss) ist das dennoch eine ganze Reihe von niederschmetternden Urteilen, die über den Technikeinsatz am Berg gefällt werden: Betrug und Selbstbetrug, Künstlichkeit, Zerstückelung der organischen Einheit, Häresie, Unterwerfung unter das technische Artefakt, Angleichung an das leblose Ding; deutlich sind dabei die Parallelen zu verschiedenen Fetischdiskursen, die vom Irrtum des Fetischisten, vom künstlichen Gott, vom zerstückelten Objekt der Begierde, von der Abgabe der Souveränität an das Objekt sprechen.

Die Rosskuppen-Dachl-Verschneidung stand, wenn schon nicht im, so doch zumindest nahe am Zentrum des Konflikts um den Einsatz technischer Mittel, wie er in den zoer und 3oer Jahren des 20 . Jahrhunderts geführt wurde. Sie trug den Beinamen >Todesverschneidung und galt einige Jahre als die schwerste Route des Gesäuses, eine zweite Durchsteigung ließ (sicher auch kriegsbedingt) bis 1947 auf sich warten. Ihre Erstbesteigung verdankt sich wie erwähnt technischen Mitteln, die zum Zeitpunkt und am Ort ihres Einsatzes alles andere als unumstritten waren: Fiechtlhaken, die als Griffe, Tritte und Umlenker für Seilzüge und Pendelquergänge dienten, Trittleitern, Sitzbretter und Klettergürtel. Elf Jahre davor war die unmittelbar benachbarte Rosskuppenkante durchstiegen worden, und auch der dort

48 Auf diese bzw. deren Begrifflichkeit greift dann mitunter der alpinistische Diskurs zurück, vgl. Schülein, Die Indianer Europas. In: ZDÖAV 1992, S. 53-60, vgl. allgemeiner kapitalismusund industrialisierungskritische Tendenzen auch bei Schmitt, Vom Gipfelkult bis zum 6. Grad. In: ZDÖAV 1962, S. 29-48; Mann, Wagnis und Ehrfurcht. In: ZDÖAV 1964, S. 174-182; Kind, Alpinismus ad absurdum? In: ZDÖAV 1998, S. 203-211.

Vgl. Lammer, Wie anders ist das Besteigen der Alpen geworden! (1937), S. 49. Einen ganz dieser Beobachtung analogen Widerspruch erwähnt auch Günther, wenn sie die häufig anzutreffende Formulierung von der >Arbeit` des Bergsteigens zitiert. Vgl. Günther, Alpine Quergänge (1998), S. 186ff., v.a. S. 195. 
vergleichsweise geringe Einsatz technischer Hilfsmittel - acht Mauerhaken - traf auf den Widerstand etwa Eduard Pichls:

Erst nach dem Kriege sind im Gesäuse einige gefährliche Stellen auf alten Wegen und ein neuer Anstieg mit Haken verunstaltet worden. Diese Zeugen eines sportlichen Fortschrittes, aber auch eines bergsteigerischen Verfalles sollten wieder entfernt werden. ${ }^{51}$

Pichl als Vertreter der >Wiener Schule schen Kletterns. Er war langjähriger Funktionär des Alpenvereins, Begründer der Austria-Jungmannschaft (intendiert als Elite der Alpenvereinssektion Austria) und trat auch im Gesäuse wirkmächtig in Erscheinung, etwa als Erstbesteiger der Planspitzen-Nordwand. Am ersten Gesäuseführer von Heinrich Heß arbeitete er ab der siebten Auflage mit, in dieser Form wurde der Führer erst in den 8oern abgelöst. Im 1927 veröffentlichten Überblicksband Wiens Bergsteigertum mokiert er sich über die aus Innsbruck und München nach Wien vorgedrungenen technischen Unsitten. ${ }^{52}$ Auf den Kronzeugen einer solchen technikkritischen Einstellung, Paul Preuß, konnte Pichl sich übrigens nicht berufen, weil er als glühender Antisemit die 1921 erfolgte Einführung des Arierparagraphen im Alpenverein betrieben hatte - die Legende Preuß jedoch (bis zu seiner Taufe 1909) Jude war. ${ }^{53}$

Rückblickend wurde mehrfach auf den Konflikt im Gesäuse - mithin ein Generationenkonflikt - Bezug genommen:

»Das letzte im Fels« zu überwinden, galt die Devise - und dazu waren schließlich auch im Gesäuse Mittel recht, die, im Wilden Kaiser schon gang und gäbe, das Cesicht konservativer Wiener nur mit Zornesröte füllten: Am 17. August 1925 trieben Karl Sixt und Fritz Hinterberger zum ersten Mal eine für damalige Verhältnisse unglaubliche Zahl von Haken - exakt acht Stück - in die Felsen der Roßkuppenkante; sogar einen Seilquergang führten sie bei ihrer Erstbegehung durch. ${ }^{54}$

Die Jugend jubelte damals, und die Alten tadelten oder schauten skeptisch in die Zukunft des Bergsteigens. Wo bleibt der Bergsteigergeist? Wenn nun auch im Cesäuse das entweihende Hämmern und das herausfordernde Singen der Mauerhaken ertönt? Götterdämmerung des Alpinismus? ${ }^{55}$

51 Pichl, Wiens Bergsteigertum (1927), S. $154 f$.

52 Diesen Unsitten stünden die Wiener ablehnend gegenüber, was sie aber, Pichl zufolge, manchmal aus Gründen der Opportunität nicht öffentlich äußerten. - Pichl, Wiens Bergsteigertum (1927), S. 140.

53 Vgl. Achreiner, Der unermüdliche Lausbub (2013), S. 12.

54 Heitzmann, Das Gesäuse. In: ZDÖAV 1988, S. 7-21, hier S. 17.

55 Maix, Menschen im Haindlkar. In: ZDÖAV 1956, S. 111-119, hier S. 111. 
Die Zeit der Zwanziger Jahre war im Gesäuse die Zeit der großen neuen Wandprobleme, die Zeit auch der - horribile dictu - ersten Mauerhaken und damit die Nach-Pichl-Zeit, von diesem daher mit vielen bösen Worten verketzert und verdammt [... ..$^{56}$

Im so beschriebenen diskursiven Umfeld ist es wenig überraschend, dass der 1937 von Raimund Schinko veröffentlichte Erstbegehungsbericht der RosskuppenDachl-Verschneidung die Bemühung zeigt, die Anwendung technischer Hilfsmittel peinlich genau zu dokumentieren und, wo möglich, zu verteidigen; Schinko zählte zu den Pionieren extremer technischer Kletterei. ${ }^{57}$

Ein Fünftel der Route, so Schinko, sei hakentechnisch zurückgelegt worden, minutiös schildert er den Wechsel von frei kletterbaren Passagen und solchen mit Hakeneinsatz. Erwähnung findet der Einsatz von Prusikschlingen im Nachstieg oder zum Wieder-Aufstieg am vorhandenen Seil sowie der von Haken und Trittschlingen an Schlüsselstellen. Aufsehenerregendstes technisches Detail ist dabei sicherlich die Überwindung einer Quergangpassage mit nur einem seichten Querriss als Haltemöglichkeit:

Solch ungewöhnliche Stellen verlangen ungewöhnliche Mittel zur Überwindung, und die haben wir mit: acht kleine Holzwürfel, welche die über das Menschenmögliche hinausgehenden Schwierigkeiten ebensogut oder schlecht wie Haken auf das eben erträgliche Maß vermindern. ${ }^{58}$

Anstatt Haken in die Wand zu schlagen, was aufgrund der Wandbeschaffenheit nicht möglich war, werden sie anscheinend mit Hilfe der Holzklötzchen in dem ansonsten zu breiten Riss festgeklemmt. Während bis hierher der Bericht - gewissermaßen im Sinne der Transparenz - Technikeinsatz nur dokumentiert, folgt auf die Holzwürfelpassage ein argumentativer Einwurf, der der damaligen Diskurslandschaft Rechnung trägt:

Sogar der Theoretiker des idealen hakenfreien Bergsports, als einzig wahrem Cefahrensport, hätte an den gefährlich schwach holzverkeilten Häkchen nichts mehr auszusetzen gehabt, die meinen Gefährten zu guter Letzt über das Unmögliche obsiegen lassen. ${ }^{59}$

So wird, rhetorisch eigenwillig, zuerst die Gleichwertigkeit von den mit Holzklötzchen fixierten und rechten , d.h. in die Wand geschlagenen Haken behauptet:

56 Baumgartner, Vom Sausen und Brausen. In: ZDÖAV 1988, S. 20-36, hier S. 30. Vgl. auch: Schwanda, Das Cesäuse (1990), S. 22.

57 Vgl. etwa Schwanda, Das Cesäuse (1990), S. 194.

58 Schinko, Neues aus dem Cesäuse (1936/1937), S. 350.

59 Ebd. 
»ebensogut oder schlecht« - Schinko nutzt die Möglichkeit, mittels der halsbrecherischen Holzwürfel auch gleich den Nutzen des normalen Hakeneinsatzes zu schmälern. Die menschliche Leistung auch beim hakenunterstützten technischen Klettern wird damit wiederum hervorgehoben.

Gleich darauf wird in einer gegenläufigen Bewegung wieder der Unterschied zwischen den beiden Sicherungsmethoden betont: Der Purist als Gegner konventionellen Hakeneinsatzes könne doch sicher zumindest der Befestigung mit Holzklötzchen zustimmen - und wenn auch sonst nicht, so doch zumindest angesichts des Siegs »über das Unmögliche« (des »Mords am Unmöglichen«, wie andere es später nennen werden). Unter Verweis auf die geringe Effektivität der Holzwürfel wird so die Bezwingung des Quergangs als gar nicht so sonderlich technisch dargestellt. Jahrzehnte später wird Fritz Sikorovsky - Schinko kommt im Zweiten Weltkrieg um - die damalige Leistung Revue passieren lassen, um sie mit späteren Begehungen derselben Route und ganz allgemein den technischen Möglichkeiten in den 7oern zu kontrastieren: »Überhaupt mit der neuzeitlichen Ausrüstung und der Hakentechnik! Man hört, liest und sieht es auch auf Bildern immer wieder: >von Haken zu Haken « ${ }^{60}$ - womit erneut der eigene damalige Technikeinsatz heruntergespielt wäre.

Über Raben thematisiert an einigen Stellen die Technikdiskussionen im Alpinismus; darüber hinaus wird ein expliziter Bezug zur Erstbesteigung der im Zentrum stehenden Route, der Rosskuppen-Dachl-Verschneidung, hergestellt, werden die Erstbesteiger als Referenzgrößen angeführt, werden ihr Bericht bzw. die Stellen aus dem Kletterführer wörtlich wiedergegeben, zur >Dom<-Passage etwa: »Wegen dieser Stelle im Bericht von Sikorowsky und Schinko und wegen jener, die den Holzklötzchenquergang beschrieb, war er vor gut zwanzig Jahren zum ersten Mal hier gewesen.« (ÜR 105f.) Ein literarischer Text, der ganz allgemein den Technikeinsatz im Alpinismus thematisiert, hat schon allein damit eine beachtliche Menge an Kontexten im Schlepptau, die genau dieses Thema mit einigem Furor und mit durchaus fetischistisch zu nennenden Werthaltungen diskutieren. Bei einem Text, der den Technikeinsatz in der Rosskuppen-Dachl-Verschneidung im Nationalpark Gesäuse zeigt, und damit sowohl auf eine Zeit als auch auf einen Ort zielt, die in der Technikdiskussion an prominenter Stelle liegen, lohnt ein näherer Blick auf den Einsatz technischer Mittel umso mehr.

60 Sikorovsky, Die Roßkuppen-Dachl-Nordverschneidung (Todesverschneidung). In: ZDÖAV 1979, S. 206-214, hier S. 206. 


\section{Technik in Über Raben}

\section{Über Raben: Technikaffirmation}

Auf die Frage, wo zwischen Dogmatismus und Pragmatismus, zwischen Technikaffirmation und Technikfeindschaft sich der Protagonist von Über Raben einordnen lässt, muss man wohl sagen: Er nimmt die Sache recht entspannt. Keineswegs hadert er damit, bei jedem seiner Schritte von Technik abhängig zu sein, von den Tourenschiern am Weg zur Hütte oder zum Wandfuß, von den Haken und Steigleitern an der Wand. Dass er in seinem Verhältnis zu Ausrüstungsgegenständen mitunter zu engen emotionalen Bindungen neigt, kam schon zur Sprache.

Hat er seine erste Begehung der Route vor zwanzig Jahren noch frei absolviert (Technik also nur zur Sicherung verwendet), so zeigt ihn der Text nun auch beim Einsatz beispielsweise von Steigleitern. »Insgesamt«, so heißt es, »konnte er [...] mit der fanatischen Haltung gewisser Leute, unter keinen Umständen ein Stück Eisen in die heiligen Berge zu treiben, nichts anfangen.«(ÜR 115) Den gängigen >Natürlichkeits-< und >Reinheitsgeboten technisch vermittelten Felskontakt vor: Ohne zu zögern verwendet er Haken und Trittleitern anstelle von natürlichen Griffen und Tritten, er verspürt »keinerlei Ehrgeiz in Richtung Rotpunktkletterei. Dafür war er zu alt und die Lage zu besonders« (ÜR 106). Hier fällt auch einer der Verweise auf die Erstbesteiger und ihren Bericht: "Die Sache bestand [...] im Wesentlichen aus dem Umhängen von Trittschlingen und Steigleitern. Er wurde Schinko und Sikorowsky nicht annähernd gerecht, aber darum ging es im Moment nicht."(ÜR 111, siehe auch 115).

In seiner Befindlichkeit wie in seinem Verhalten unterstreicht Schneider oft noch die technisch verursachte Distanz zur Sphäre des Natürlichen: Während Technik anderswo als Hindernis des Naturerlebnisses wahrgenommen wird (ein Topos unter alpinistischen Technikkritikern wie Lammer ${ }^{61}$ ), bietet ihm gerade die Konfrontation beider Sphären ein Moment ästhetischen Genusses: »Er konnte den Ton nachsingen, den der Eisschild von sich gegeben hatte, als Sepp Stadler den einzigen langen Eishaken, den sie bei sich hatten, in die Wand donnerte«, heißt es etwa, oder: »Er liebte das leise Zischen der Felle und die Art, in der sich der Schnee vor den Schispitzen zusammenschob, bei jedem Schritt« (ÜR 55, 14f.).

Am oben erwähnten Quergang mit Holzwürfelchen (der auch in späteren Gesäuse-Kletterführern noch »Holzpackelquergang« heiß $\mathrm{t}^{62}$ ) zeigt sich, dass ihn

61 »[K]aum daß er den Fels berührt mit Fuß und Hand. Nur noch mit Ringnagel, Hammer, Schnapper und Seil hat er zu schaffen, nur noch nach einer Ritze für den Stift schaut er den Fels an.«-Lammer, Wie anders ist das Besteigen der Alpen geworden! (1937), S. 33.

Vgl. etwa: Schall, Wagner u. Wolf, Kletterführer Gesäuse (1984), S. 39. 
neben seiner geradezu alltagspraktischen Herangehensweise und dem ästhetischen Genuss an der Technik noch anderes von den Phantasmen alpinistischer >Ursprünglichkeit< trennt: Er begegnet der Route nicht als einer vermeintlich natürlichen Gegebenheit, sondern als einem historisch geformten, quasi-kulturellen Artefakt. Explizit erstreckt sich seine Begeisterung für das Bergsteigen auch auf dessen verschriftlichte Formen. Die Texte aus dem alpinistischen Diskurs, die Routenbeschreibung, aber auch die umfangreiche Diskussion zum Technikeinsatz, werden hier - ganz analog zum Status, der der Psychoanalyse bei Hochgatterer zukommt - nicht nur auf ihren faktualen Gehalt hin befragt, sondern stellen für den Protagonisten wie für den Text kulturell wirkmächtiges Material dar, mit dessen Motiven kreativ umgegangen werden kann. Im Herbst vor Einsetzen der Handlung hat der Protagonist in der Route ein >re-enactment tradierten Vergangenheit inszeniert: »Aus historischer Sentimentalität hatte er damals einige eigens zugerichtete Holzklötze in den Riss geklopft. Bernhard [sein Seilpartner, Anm.] hatte gegrinst, aber nichts dagegen gesagt.« Und als der Protagonist nun wieder an den selbstgesetzten Holzklötzen vorbeikommt:

Die Holzklötze waren wenigstens noch da, im Riss festgefroren und in ihrer Nutzlosigkeit ausschließlich dekorativ. Er hatte sechs flache Quader aus drei verschiedenen Holzarten zugeschnitten gehabt, Birke, Buche und Akazie, je zwei Stück. Es war tatsächlich ziemlich bescheuert gewesen. (ÜR 111)

Was ist das also für ein Wesen, das sich hier den Berg hinaufbewegt? Wäre es nicht auf vielfältige Weise von Ausrüstungsgegenständen umgeben, könnte es natürlich den Weg, den es zurücklegt, nicht gehen. In ziemlich jeder Umgebung ist es von materiellen Gegenständen abhängig, um Witterungseinflüssen zu widerstehen, sich fortzubewegen und zu orientieren, Nahrung zu beschaffen und zuzubereiten und so fort. Schneider allein, das heißt: ohne jegliche Artefakte, wäre ein äußerst verletzlicher Organismus, weder zur Fortbewegung im Schnee und am Fels, noch zur Nahrungsbeschaffung, noch für die Kälte und den Wind ausgerüstet. Er jedoch ist im Reinen mit der eigenen unzureichenden biologischen Angepasstheit an die Umgebung und den Möglichkeiten bzw. Notwendigkeiten, diesem Mangel technisch zu begegnen. Er ist ein zufriedenes bio-technisches Wesen, eine spezifisch auf den Winteralpinismus ausgerichtete Mischung aus Mensch und Werkzeug. Eine solchermaßen ausgewogene, geradezu geläuterte Haltung allerdings wird vom Verlauf des Textes widerlegt.

Mit der Figur der technisch gemeisterten Ausgesetztheit ist ein den Text auf ganz basaler Ebene grundierender Kontext aufgerufen, ein Kontext gewissermaßen jedes affirmativen Technikbezugs: das Bild der Technik als Ergänzung und Verlängerung des Menschen, wie es prominent in der philosophischen Anthropologie geprägt wurde. Technikgebrauch steht hier vor dem Hintergrund der grundlegenden Unangepasstheit des Menschen an die Natur und der daraus resultierenden 
Notwendigkeiten. Der Mensch ist, wie Arnold Gehlen in Anlehnung an Herder formuliert, ein >Mängelwesen<, das, »der rohen Natur wie ein Tier ausgesetzt, mit seiner ihm angeborenen Physis und seiner Instinktmangelhaftigkeit unter allen Umständen lebensunfähig « ${ }^{63}$ sei. Daraus resultiert die Notwendigkeit der technischen Unterwerfung der Natur, des »in die Welt hineingebaute[n] Nest[s]. ${ }^{64}$ Technik macht die >organische Mittellosigkeit « wett, durch »Organersatz, Organentlastung, Organüberbietung ${ }^{65}$ - so die bei Gehlen häufig anzutreffende Trias. Gerade im Fall des Alpinismus scheint das besonders naheliegend, wie man beispielsweise dieser Passage bei Aufmuth entnehmen kann:

Wir nehmen Daunenjacken und Biwaksäcke mit, um dem Erfrierungstod vorzubeugen; wir versehen uns mit Spezialcremes, damit die Haut nicht verbrennt, wir tragen Spezialbrillen, damit die Augen nicht erblinden, wir packen Steigeisen und Pickel ein, um nicht abzugleiten, Seile, um nicht abzustürzen, wir haben Schmerztabletten dabei und üben uns im Abtransport von Schwerverletzten. ${ }^{66}$

Jeder Aspekt des Verweilens und der Bewegung im Gebirge braucht sein technisches Komplement. Dementsprechend listet hier Aufmuth die Dinge, die in den Bergen notwendig sind, um die organische Mittellosigkeit des Menschen auszugleichen, und dementsprechend lang sind die erwähnten Packlisten in Über Raben ebenso wie in der alpinistischen Ratgeberliteratur. Ein Technikverständnis, wie es die philosophische Anthropologie liefert, ist grundsätzlich affirmativ: Die Entwicklung der Technik ist hier logische Folge der Tatsache, dass der Mensch eben für keine - eben auch nicht für die alpine - Umwelt natürlich ausgestattet ist. Auch im alpinistischen Kontext sind explizite Hinweise auf die philosophische Anthropologie gerade dort zu finden, wo technikkritische Standpunkte relativiert werden. ${ }^{67}$

Soweit könnte man dem Roman eine technikaffirmative Position unterstellen. In Schneiders undogmatischer, ästhetisch genießender Haltung ist Kletterpuris-

63 Gehlen, Zur Geschichte der Anthropologie (1986 [1957]), S. 17f. Vgl. u.a. auch ders., Ein Bild vom Menschen (1986 [1942]), S. 46; ders., Die Technik in der Sichtweise der Anthropologie (1986 [1953]), S. 93f. Fingerzeige hierzu verdanke ich der materialreichen Geschichte der Techniktheorien von Susanne Fohler: Fohler, Techniktheorien (2003).

64 Gehlen, Zur Geschichte der Anthropologie (1986 [1957]), S. 21.

65 Ders., Die Technik in der Sichtweise der Anthropologie (1986 [1953]), S. 93; ders.: Sozialpsychologie (1986 [1949/1957]), S. 148.

66 Aufmuth, Zur Psychologie des Bergsteigens (1988 [1984]), S. 14.

67 Vgl. z.B. Karl Greitbauer, der unter explizitem Bezug auf Gehlen die Existenz eines >Unbehagens an der Technik in Zweifel zieht und Bergsteigen als Äußerung des >expansiven Ceistesく mit der industriellen Entwicklung parallel setzt. - Greitbauer, Wohltemperierte Gesellschaft. In: ZDÖAV 1963, S. 176-183, hier S. 178. Lenk (»Den wirklichen Gipfel werde ich nie erreichen«. In: ZDÖAV 1985, S. 103-114) sieht unter Bezug auf Plessner und Scheler Alpinismus zu sehr von den Normen der industriellen Cesellschaft geprägt, um »gesellschaftlicher Totalprotest « (S. 113) sein zu können. 
mus nur ein vorübergehendes Moment, das im Fall des Falls dem Technikeinsatz weicht. In Gehlens >technischem Nest « fühlt er sich ganz wohl. Gelesen in einem technikaffirmativen Kontext wie der philosophischen Anthropologie ergibt sich der Eindruck eines souverän über seine Artefakte gebietenden Subjekts im Akt der Naturunterwerfung, einverstanden mit den Notwendigkeiten von Organersatz, -entlastung und -überbietung.

Das Problem mit dieser Lesart liegt im Ende der Gesäuse-Kapitel von Über Raben. Die abschließende Katastrophe und mehrere Gesichtspunkte am Weg dorthin lassen eine affirmative Lesart des Verhältnisses Mensch-Ding und eine Deutung des menschlichen Handelns als auch unter technischen Bedingungen selbstbestimmt nicht $\mathrm{zu}$; zahlreiche Momente in seinem Verhältnis zu den Dingen unterlaufen die Souveränität des Protagonisten.

\section{Technikkritik I: Abhängigkeit}

Dem ungenierten Einsatz technischer Hilfsmittel im Alpinismus sowie in Über Raben und der affirmativen Haltung dazu steht innerhalb des Diskurses erwähntermaßen einiges entgegen: Die logische Kehrseite von Technik als Behebung eines Mangels ist die in den Menschen hineinwirkende, abhängig machende Technik wie sie konkret etwa bei Lammer moniert wird; die säuberliche Trennung von souveränem Subjekt und bloß dienendem Objekt würde hier untergraben, die Positionen mitunter vertauscht. ${ }^{68}$

Umso schwerwiegender wird hier ein weiterer obligatorischer Aspekt des Technikeinsatzes - der technische Defekt: »Die Erfindung des Schiffes war gleichbedeutend mit der Erfindung des Schiffbruchs, die Erfindung der Dampfmaschine, der Lokomotive schloß die Erfindung der Entgleisung, des Eisenbahnunglücks mit ein« wie Paul Virilio diese Nachtseite der philosophischen Anthropologie formuliert. »Die Errungenschaft eines Werkzeugs, eines neuen industriellen oder für welchen Zweck auch immer bestimmten Instruments bedeutet immer zugleich auch die

68 Gehlen spricht angesichts dieser existenziellen Abhängigkeit nicht von der Gefährdung einer Essenz des Humanen durch Technik (wie das in technikkritischen Anmerkungen der Fall ist), ganz im Gegenteil: Nicht nur der einzelne Mensch, sondern Menschlichkeit an sich ist nur im Zusammenspiel mit dem technischen Artefakt denkbar, in Gehlens Begriff von »Menschlichkeit« wird diese geradezu zur Begleiterscheinung der Technik. Die Notwendigkeit, sich ihrer zu bedienen, wird Gehlen zum Anthropologikum schlechthin: »Die Technik ist so alt wie der Mensch« (Gehlen, Die Technik in der Sichtweise der Anthropologie (1986 [1953]), S. 93) - damit aber auch, kann man einwenden, dieser so alt wie jene. Dieser Begriff von Menschlichkeit ist also, ebenso wie der einzelne Mensch, abhängig von Technik. Der weitere Verlauf der Passage wirft Fragen auf: „Der Beweis läßt sich daran führen, daß wir umgekehrt erst aus Spuren der Werkzeugbenutzung mit Sicherheit schließen können, daß wir es mit Menschen zu tun haben« (ebd.) - was entweder ein Zirkelschluss ist oder eine Definition des Wortes Mensch. 
Errungenschaft einer Gefahr, eines speziellen Risikos « ${ }^{69}$ - und dieses ist natürlich umso größer, je nützlicher das technische Ding davor war:

Er erinnerte sich an jenen Jännersonntagmorgen, an dem ihm in der Ostwand des Piz Badile der Hammerkopf zersprungen war. Er erinnerte sich an den hellen, ewig nachsingenden Ton, daran, wie sein Blick dem in weitem Bogen abfliegenden Stahlfragment gefolgt war und wie es ihm schließlich dieses lange Lachen herausgestoßen hat, ein Lachen, das vollkommen in der Einsamkeit lag und ganz nah am Tod. »Erfrieren oder abstürzen?«, hatte er gedacht. (ÜR 17f.)

Von Beginn an ist der Defekt stilles, gegen Ende immer weniger stilles Nebenthema in Über Raben, und bei entsprechender Lektüre wird deutlich, wie häufig im Umgang mit den Dingen das Fehlfunktionieren eben dieser Dinge eine Rolle spielt, das Misslingen, die Verletzung, der Defekt: Der Schistock macht besorgniserregende Geräusche, der Schuh hat eine Schnalle zu wenig (ÜR 10f., 17), die Bindung ist bei Kälte schwergängig (ÜR 56), das Gewehr zieht nach links unten (ÜR 16). Hammerköpfe splittern (s.o.), die Steigeisen (Lammers Verschmelzungsphantasien zum Hohn) passen nicht, ein anderes Paar Steigeisen spielt die Hauptrolle in der Erinnerung an eine schwere Kopfverletzung (ÜR 157, 116).

Ein Höhepunkt des technischen Versagens hat eine ganz unauffällige Vorgeschichte, einen kleinen, harmlos scheinenden Schnitt an der Innenseite des rechten Mittelfingers, »vom Kalkfels und vom Frost « (ÜR 114). Dieser führt zu immer schwereren Symptomen: Die Hand kühlt ab, Zeige-, Mittel- und Ringfinger schwellen an, später ist der Unterarm, dann der Arm bis zur Schulter betroffen (ÜR 165, 200f., 211, 229). ${ }^{70}$ Die fatale Konfrontation von Mensch und Technik, Organischem und Anorganischem zeigt sich nun schon auf vorerst harmlose Art und Weise, in der Tatsache, dass am anschwellenden Finger der Ehering feststeckt (ÜR 201), dessen Entfernung eine einigermaßen grausige technische Prozedur erfordert:

Er schluckte zwei Parkemed und trank den Flachmann leer. [...] Er schnitt von einer Reepschnur ein knapp einen Meter langes Stück ab, schlitzte den Mantel auf und isolierte aus dem Kern ein Bündel weiß glänzender Kunststofffasern. Mit Hilfe von Speichel drehte er es zu einem dünnen Faden. An der Kuppe beginnend, führte er ihn in engen und möglichst festen Windungen um den Finger herum, langsam und sorgfältig. Er quetschte auf diese Weise die Schwellung sukzessive aus dem Finger hinaus. [...] Die Sache tat höllisch weh, trotz der Tabletten, die er

69 Virilio, Das Pannenmuseum (1998 [1986]), S. 93, 94.

70 Eine laienhafte Internetrecherche lässt mich die Diagnose »Phlegmone in den Raum stellen, eine oft durch eine kleine Hautverletzung ausgelöste, sich im Bindegewebe ausbreitende eitrige Infektionserkrankung. 
genommen hatte. Als er an der Schnürfurche angelangt war, fettete er die Fadenspirale mit Hirschtalg ein und zog den Ring unter behutsamem Drehen ab. (ÜR 207f.)

Konnte hier einer technischen Bedrohung (dem abquetschenden Ring) noch technisch begegnet werden, so tritt die Technik Schneider vor seinem Tod, seinem Sturz aus der Höhle, nicht mehr helfend zur Seite. Im Gegenteil: Von der schmerzstillenden Wirkung einer Mischung aus Codein und Alkohol betäubt und behindert von seiner mittlerweile unbrauchbaren rechten Hand, wird er zum wehrlosen Opfer der >Tücke des Objekts`: Der Topf kippt, der Gaskocher steht schief, der Anzünder verweigert den Dienst. Er bleibt am Packsack hängen, das Fernglas ist zu lichtschwach, der Blick durch das Zielfernrohr verschwimmt (ÜR 230ff.). Beim abschließenden Sturz aus der Höhle richtet sich seine letzte Wahrnehmung auf den Raben, der schon seit Tagen an seinem Nest gebaut, es letztendlich auch fertiggestellt und in Gebrauch genommen hat und in diesem Moment geradezu als Triumph der Natur über den technisch erweiterten Menschen gelesen werden kann; das technische Nest ist dem natürlichen (es »sah richtig nett aus, sogar einige kleine schwarze Federn gab es als Polsterung « ÜR 230) unterlegen.

Schneider ist den Dingen ausgeliefert. Versagen sie ihm den Dienst, ist er in dem Maß verloren, wie er davor von ihnen profitiert hat; gerade an der nur technisch begehbaren Felswand wird das deutlich. Alpinistische Technik ist zum Gutteil solche, die den Absturz verhindern soll, und gerade diesen Dienst versagt sie ihm am Ende des Romans. Die beobachtbare Häufung macht den Eindruck, als würde hier der Unwillen des alpinistischen Artefakts inszeniert. Während Schneider mit den Beschädigungen seiner Ausrüstung zu leben gewohnt war (dem Schuh, dem Stock), versagen die Dinge ihre Gefolgschaft in dem Moment, in dem Schneiders eigene Funktionstüchtigkeit nachlässt. Das Verhältnis des Alpinisten - und Schneiders im Speziellen - zur Technik entspricht angesichts dieser bedrückenden Evidenz tatsächlich den Schreckensbildern von Abhängigkeit und Unterwerfung, wie sie Ziak, Lammer und Co. malen.

\section{Technikkritik II: Unterwerfung und Mortifizierung}

Damit ist also der Kontext eines affirmativen Technikbegriffes als Folie für die Lektüre von Über Raben unzureichend: Technik ist hier nicht bloß Werkzeug und behelligt das Subjekt ansonsten nicht weiter, sie stellt stattdessen nicht nur eine Erweiterung, sondern auch eine Bedrohung des Subjekts dar. Diese Bedrohung liegt nicht nur in den Auswirkungen des konkreten Artefakts, sondern auch im sSystem` Technik, das, nachdem es den Alltag zur Gänze affiziert hat, nun auch in die Berge vordringt. Nicht zufällig stammen die Gegenbilder, mit denen Lammer unwürdiges technisches Klettern abqualifiziert, aus dem handwerklichen und in- 
dustriellen Bereich: ${ }^{71}$ Dem Diskurs des Alpinismus eignet, wie sich gezeigt hat, eine gesellschafts- und modernisierungskritische Tendenz, die den Berg als Gegenwelt zum >Reich der Zwecke bewahrt wissen will und mit Schaudern den Einzug des Effizienzdenkens in die Berge registriert, was nicht zuletzt mit dem Begriff des Sports, das heißt mit Messbarkeit und Leistungsdenken zusammenhängt. ${ }^{72}$ In dieser Hinsicht gibt es also gewissermaßen immer schon eine >Kritische Theorie des Alpinismus ${ }^{73}$, und nicht zufällig finden sich auch im alpinistischen Diskurs später die Begrifflichkeiten der Kritischen Theorie wieder.

Bei Horkheimer und Adorno wurden bekanntlich diese technikkritischen Einwürfe wirkmächtig formuliert, mit fast durchgehend hohem Abstraktionsgrad allerdings und der entsprechenden Distanz zum konkreten Artefakt: ${ }^{74}$ Technikkritik ist hier Kritik an Technik als übergreifendem System, an den gesellschaftlichen Auswirkungen von Wissenschaft und Industrialisierung. Der Fokus liegt dabei auf der technischen Rationalität, der nach Horkheimer sinstrumentellen Vernunft ${ }^{75}$, die sich zunehmend auf alle gesellschaftlichen Bereiche ausbreitet, den Menschen ihren Maßstäben unterwirft und seine Denk- und Verhaltensweisen determiniert, wobei die technische Ratio dabei auch in politische Machtstrukturen umschlägt, die »Maschine $«$ in $»$ Herrschaftsmaschinerie ${ }^{76}{ }^{76}$. Menschliches Handeln muss nicht vom konkreten technischen Werkzeug gestützt sein, um als technisch zu gelten: Technisch ist es, sobald es den Maßstäben von Zweckorientierung, Leistung und Effizienz entspricht; Technik erschaffe »technologische, auf Technik eingestimmte Menschen ${ }^{77}$.

Kernpunkt dieser >Dämonie der Technik ist die Objektifizierung des Menschen nach technischer Maßgabe - auch über den Umweg des Sports - , wie sie in der Dialektik der Aufklärung und anderswo aufgezeigt wird. Das Verhältnis der Gesellschaft zur Technik sei so doppeldeutig wie das zum Sport, zudem seien beide verwandt,

71 Vgl. etwa Lammer, Wie anders ist das Besteigen der Alpen geworden! (1937), S. 34.

72 Dementsprechend treffen sich die Kritische Theorie und ein Teil des alpinistischen Diskurses auch hinsichtlich ihrer Bewertung des Sportgedankens. Mit der Stoppuhr am Berg überschreite man die Grenze »vom Erhabenen zum Lächerlichen«, als individuelle Tätigkeit gebe es beim Bergsteigen »nichts zu messen und zu stoppen«. - Ziak, Der Mensch und die Berge (1936), S. 182; Jennewein, Vom Prinzip der Auslese. In: ZDÖAV 1954, S. 5-12, hier S. 8.

Der Topos von der Unterwerfung aller Lebensbereiche - und schließlich des Menschen selbst - wird zeitgenössisch und in den darauffolgenden Jahrzehnten intensiv diskutiert, etwa bei Hans Freyer, Carl Schmitt, Ernst ]ünger, Oswald Spengler, Friedrich Georg Jünger, Günther Anders, Jacques Ellul. Vgl. Fohler, Techniktheorien (2003). 
so Adorno. ${ }^{78}$ >Sport< sei als Unterkategorie von >Freizeit< ohnehin notwendiges Gegenstück und damit Verlängerung der Arbeit; ${ }^{79}$ in ihm werde der menschliche Körper einem funktionalistischen Blick unterzogen, der zugleich seine Unterwerfung, Zerstückelung und Mortifizierung in sich trage; einem funktionalistischen Blick, der den Systemen von Faschismus und Kapitalismus gleichermaßen eigne. Sport »Fitness in ihrer fetischisierten Form « - sei Angleichung an die Maschine: Die Verfechter des Sports sehen »den Körper als beweglichen Mechanismus, die Teile in ihren Gelenken, das Fleisch als Polsterung des Skeletts. Sie gehen mit dem Körper um, hantieren mit seinen Gliedern, als wären sie schon abgetrennt $"{ }^{80}$ - der Leib unter den Voraussetzungen der Technik wie des Sports erfährt eine >Transformation ins Tote $<$, der ertüchtigte Körper wird zur Leiche. ${ }^{81}$

Aus einer technikaffirmativen Perspektive wie der Gehlens kommt man unversehens zum selben Schluss - freilich unter anderen Vorzeichen: Gehlen zufolge ist

der Mensch in der Tat in ganz zentralen Bereichen seiner Natur Automatismus, er ist Herzschlag und Atmung, er lebt geradezu in und von sinnvoll funktionierenden, rhythmischen Automatismen, wie sie in der Bewegung des Cehens, vor allem aber in den eigentlichen Hantierungen und Arbeitsgängen der Hand vorliegen, in dem >Handlungskreis`, der über Sache, Hand und Auge zur Sache zurücklaufend sich schließt und dauernd wiederholt. ${ }^{82}$

Dementsprechend wird ihm Technik zum Vehikel menschlicher Selbsterkenntnis: Die Begeisterung des Menschen durch den technischen Automatismus - der »Zauber [...], den die Autos auf unsere Jugend ausüben ${ }^{83}$ und ähnliches - ist auf seine Ähnlichkeit mit dem menschlichen Organismus zurückzuführen. Indem Technik die Gelegenheit bietet, Bewegung außerhalb des eigenen Körpers wahrzunehmen, bietet sie Einblick in die menschliche Natur; Technik und Mensch entsprechen einander: Im Anblick des bewegten technischen Artefakts »schwingt etwas in uns mit,

78 Vgl. ebd., S. 685. Hinweise zum Verhältnis der Kritischen Theorie zum Sport verdanke ich Vaz, Sport und Sportkritik im Kultur- und Zivilisationsprozess (2004).

79 »Im Zeitalter wahrhaft beispielloser sozialer Integration fällt es schwer, überhaupt auszumachen, was an den Menschen anders wäre als funktionsbestimmt. Das wiegt schwer für die Frage nach der Freizeit.«-Adorno, Freizeit (1996 [geh. 1969]), S. 645.

Horkheimer u. Adorno, Dialektik der Aufklärung (1996 [1947]), S. 269. Dieses Diktum bezieht sich in diesem Fall auf den Sportkult derer »drüben«, d.h. der Faschisten auf dem europäischen Kontinent; das Verdikt zum Sport erhält im Werkkontext allerdings allgemeine Cültigkeit.

81 Vgl. ebd., S. $268 f$.

82 Gehlen, Sozialpsychologie (1986 [1949/1957]), S. 157, vgl. S. 161.

83 Ebd., S. 156. 
gibt es eine Resonanz in uns, und wir verstehen begrifflos und wortlos etwas von unserem eigenen Wesen. ${ }^{84}$

Hier wird der Grundstein für eine Perspektive gelegt, aus der technischer und organischer Apparat letztlich ununterscheidbar werden. Aus einer Perspektive, in der die technische nahtlos an die menschliche Ausstattung anschließt und analog funktioniert, verschwimmt die Grenze zwischen Mensch und Artefakt. ${ }^{85}$

Die Homogenisierung von Organismus und Mechanismus wird durch viele Momente in Über Raben nahegelegt. Technik und Körper erfahren die gleiche Behandlung: Wie das Seil auf Schwachstellen kontrolliert wird, unterliegen Muskulatur und Gelenke steter Beobachtung auf ihre Funktionstüchtigkeit hin. Die Defekte verteilen sich im Text dementsprechend gleichmäßig auf das Konglomerat aus Körper und Artefakt, das Schneider darstellt. Die Abgrenzung zwischen fühllosem, funktionalem Objekt und dem als Teil des Selbst empfundenen, fühlenden Körperglied ist alles andere als trennscharf. Schon zum Schmerz, dem vermeintlich sichersten Kriterium von Körperlichkeit, hat Schneider »eine eigene Beziehung«: Weil er gegen ihn in mancher Hinsicht unempfindlich ist, vermutet ein Zahnarzt in ihm entweder einen Yogi oder ein neurologisches Phänomen (ÜR 12). Beunruhigung geht für Schneider dafür gleichermaßen vom drohenden Versagen des Stocks aus wie von dem des Kieferknochens (der für ein Implantat nicht tragfähig genug sein könnte, ÜR 12). Dass der Stock ein seltsames Geräusch von sich gibt und die Schnalle eines Schuhs defekt ist, affiziert weniger die technische Funktionalität als vielmehr die Psyche des Protagonisten (ÜR 1of.); eine schwergängige Bindung (ÜR 56) entspricht spiegelbildlich dem lädierten Knie. Schließlich gipfeln all diese Defekte im alkohol- und opiatinduzierten psychischen shut down<, gefolgt von der körperlichen Auslöschung. Schon Schneiders Lösungsansatz für das Problem seines über dem Ehering anschwellenden Fingers war, wie alternativlos auch immer, ein ausgesprochen technischer: Die Zurichtung des Organismus zum

84 Gehlen, Die Technik in der Sichtweise der Anthropologie (1986 [1953]), S. 97. Von der verbreiteten abwertenden Rede über die Technik als >bloße Ratio < und >bloße Nützlichkeit « distanziert sich Gehlen. Das Unbehagen an der Technik, das auch er konstatiert, führt er darauf zurück, dass die Technik regionalen, kulturellen und nationalen Kategorien gegenüber indifferent ist, dass sie nicht Völker, sondern die Menschheit zum Subjekt wählt (was aber tatsächlich, so Gehlen, ihr quasi völkerverbindendes Potenzial ausmache). Vgl. ebd., S. 101 u.ö.

85 Siehe dazu eine technikphilosophisch informierte Anmerkung aus einem Zeitungskommentar Hochgatterers (anlässlich des 11. September 2001): Anstelle der Beherrschung der Dinge stehe gegenwärtig vielmehr eine »bedienungspartnerschaftliche Beziehung«, die »Phänomene der Grenzverwischung, also der Verschmelzung « mit sich bringt. »Letztlich greift umfassende Identifikation Platz und damit ein Prozess der Dingwerdung, der Reifizierung des Menschen selbst. [...] Das selbstgewisse, selbstmächtige und ganzheitliche Subjekt ist tot.« - Hochgatterer, Tot gestellt (2002), S. 38f. 
fühllosen >Werkstück` als Voraussetzung jedes medizinischen Eingriffs wird hier, indem es sich um eine Selbst-Zurichtung handelt, noch unterstrichen.

Bringt man die Reihenfolge der Defekte in Anschlag, könnte man geradezu meinen: Die optimistischen Techniktheorien und die dort vorliegenden Entwicklungskonzepte finden hier ihre höhnische Zurückweisung. Die philosophische Anthropologie hatte die historische Abfolge technischer Entwicklungen als eine von außen nach innen, von der Peripherie zum Zentrum des Körpers imaginiert. Die Objektivationen menschlicher Organe und Fähigkeiten würden (so zitiert Gehlen Hermann Schmidt) von außen nach innen fortschreiten, zuerst würden nur »Organleistungen verstärkt, übertrumpft, verbessert und entlastet«, dann »Kraftleistung [...] der unbelebten Natur aufgebürdet « und in einem dritten, von Gehlen in seiner aktuellen Gegenwart beobachteten Schritt auch die zerebralen Akte von Steuerung und Kontrolle nach außen verlegt, wobei Gehlen auf die überlegenen Fähigkeiten etwa von Rechenmaschinen verweist; ein Rückgriff auf diese These findet sich auch bei Habermas. ${ }^{86}$

Eine Abfolge von außen nach innen - von der Gerätschaft über die Gliedmaßen bis zum Gehirn - zeigt sich nun auch in Über Raben. Was sich hier allerdings mit derselben Bewegungsrichtung abspielt, ist eine Kette an Defekten. ${ }^{87}$ Indem sie die vorliegende Mensch-Maschine heimsuchen und dabei von den technischen Verlängerungen der »Funktionen des Bewegungsapparates« zum »steuernden Zentrum[] « fortschreiten (wenn auch unter Auslassung der »Kraftleistung«), wird aus der Entwicklung des technischen Fortschritts die Abwicklung technischen Versagens.

Auch der Widerstreit zwischen dem zweckfreien Raum der Berge und seiner technisch-rationalen Kontamination, zwischen der Abwendung des Alpinisten vom Alltag und seiner gleichzeitigen Unterwerfung unter dessen Maßstäbe lässt sich in Über Raben nachvollziehen: Schneiders alpine Leidenschaft dient als Gegenwelt zum >Reich der Zwecke - eine Gegenwelt, der er jetzt umso dringender bedarf, als er aus dem Flachland flieht. Auch in seinem Fall steht der Alpinismus also gleich

86 Gehlen, Sozialpsychologie (1986 [1949/1957]), S. 160; die Stelle bei Habermas lautet: »Zuerst sind die Funktionen des Bewegungsapparats (Hände und Beine) verstärkt und ersetzt worden, dann die Energieerzeugung (des menschlichen Körpers), dann die Funktionen des Sinnesapparates (Augen, Ohren, Haut) und schließlich die Funktionen des steuernden Zentrums (des Gehirns).«-Habermas, Technik und Wissenschaft als >Ideologie`(1969), S. 56; vgl. Fohler, Techniktheorien (2003), S. 8of.

87 Der chronologische Verlauf der Defekte, soweit sich das feststellen lässt (wobei hier eine Schuppenflechte und ein fehlender Zahn aufgrund ihrer Folgenlosigkeit unberücksichtigt bleiben), ist der folgende: Stock, Schuh und Bindung in unbestimmter Vorzeit, das Knie als rekurrentes Phänomen, das aktuell aber immerhin nach den Defekten in den zuvor genannten Utensilien auftritt, daraufhin die Hand, infolgedessen der Unterarm, die Schulter, zum Abschluss die Augen und das Gehirn. 
zweimal im Zeichen einer Alltagsflucht, nicht unpassend auch mit dem Signum der Devianz versehen. Zugleich jedoch wird der Alpenraum beide Male einem Zweck unterworfen, der auf unterschiedliche Weise den genannten »Anforderungen der Arbeitswelt« geschuldet ist: indem er erstens dem sportlichen Ausgleich und zweitens, später, der Flucht dient (bezeichnenderweise wähnt er auch die Kolleginnen und Kollegen aus dem Lehrerzimmer unter seinen Verfolgern).

Auch in ihren Details weist seine Tätigkeit den Zwiespalt zwischen der Abwehr alltäglicher Ratio und ihrer Verlängerung in den Alpinismus auf. Angesichts seines vor körperlicher Belastung bevorzugten Nahrungsmittels heißt es:

Den Schülern erzählte er nie etwas von Neapolitaner-Waffeln. Kohlehydrate vor körperlicher Betätigung, das ja, aber Kohlehydrate, das hieß Traubenzucker und Müsli und Dörrobst, all jene Dinge, von denen man in den Lifestyle-Magazinen lesen konnte und die man auch in den Lehrveranstaltungen über Sportmedizin und Ernährungsphysiologie eingetrichtert bekommen hatte. (ÜR 56)

Zwar findet hier eine dem Typus des Alpinisten entsprechende Abwehr von Vernunftgründen zugunsten eigener Erfahrungswerte und Werthaltungen (»der wunderbaren roten Schrift auf dem durchsichtigen Zellophan« - ÜR 56) statt. Dennoch unterliegen natürlich seine Ernährungsgewohnheiten letztlich dem Effizienzdenken (die Waffeln fungieren als Zuckerlieferant). Überhaupt lässt sich an Schneiders Behandlung des eigenen Körpers der Maßstab einer technischen, instrumentellen Ratio ablesen: Um die Leistungsfähigkeit aufrecht zu erhalten, wird die Ernährung der Belastung angepasst, werden ständig isometrische Übungen gemacht, Magnesiumtabletten eingenommen, Gelenke >durchbewegt und so fort (ÜR 11f., 55f., 110, 113, 153, 201 u.ö.). Am deutlichsten zeigt sich das, was aus Sicht der Kritischen Theorie unter Fragmentierung und Objektifizierung des eigenen Körpers fallen muss, am Umgang mit genannten Defekten. Wo der Protagonist kann, behandelt er sie - mit Dehnübungen, Kühlung und Schonhaltungen für das Knie (ÜR 14, 51 u.ö.) sowie der erwähnten Maßnahme gegen den anschwellenden Finger - oder zumindest das aus ihnen resultierende Schmerzempfinden, mit einiger Routine: "Stufe eins: zwei Tabletten Parkemed fünfhundert (ÜR 21 u.ö.). Körpertechniken haben hier den Charakter von technischen Instandhaltungsmaßnahmen, der Körper wird zur Gerätschaft, zum tendenziell fühllosen, zweckorientierten, vielgliedrigen Mechanismus. Eine affirmative Haltung dazu, wie sie noch die Rede vom >Mängelwesen $<$ nahelegt, wird vom Text - mit Blick auf das Ende - nicht mehr gedeckt. Und womöglich lässt sich sogar der Tod Schneiders hier nicht als unglücklicher Zufall, sondern als logische Folge der Angleichung seines Körpers an das Leblose schon zu Lebzeiten lesen; in konsequenter Durchführung von Adornos "Transformation ins Tote 


\section{Technikkritik III: Dingliche Kontamination menschlichen Handelns}

Unumwunden meint Gehlen zur technischen Determiniertheit menschlicher Handlungen: "Die Technik umgibt also nicht nur den modernen Menschen, sie dringt in sein Blut ein. ${ }^{88}$ - "Man wird dem neuen Menschentypus nicht gerecht ohne das Bewußtsein davon, was ihm unablässig, bis in die geheimsten Innervationen hinein, von den Dingen der Umwelt widerfährt « ${ }^{89}$, so wiederum Adorno, erneut dasselbe mit gänzlich anderer Werthaltung feststellend. Festgestellt bzw. angekreidet wird das Hineinagieren technischer Artefakte in Handlungen und Intentionalität, die bisher vermeintlich rein menschliche Angelegenheiten waren ${ }^{90}$. In den Minima Moralia, in einer der wenigen Passagen Adornos, die einen konkreten technischen Vorgang zum Thema haben - das Schließen von Türen -, stellt er fest, wie die Apparate (also Auto- und Kühlschranktüren, selbstschließende Türen, Türen mit Knäufen statt Klinken) menschliches Handeln nachhaltig beeinträchtigen: »In den Bewegungen, welche die Maschinen von den sie Bedienenden verlangen, liegt schon das Gewaltsame, Zuschlagende, stoßweis Unaufhörliche der faschistischen Mißhandlungen «; auch verführe etwa das Auto schon kraft der Kraft seines Motors dazu, »das Ungeziefer der Straße, Passanten, Kinder und Radfahrer, zuschanden zu fahren ${ }^{91}$. Die Artefakte, so Adorno, geben ihre Handhabung vor, und nachdem die modernen, der technischen Ratio gehorchenden Artefakte die Möglichkeiten dieser Handhabung auf das rein instrumentelle beschränken, »ohne einen Überschuß, sei's an Freiheit des Verhaltens, sei's an Selbständigkeit des Dinges zu dulden ${ }^{92}$, verrohe der Mensch im Umgang mit ihnen, indem er sich ihnen angleicht.

Deutlich scheint hier eine Romantik des Ursprünglichen durch; Schreckensbild ist eine den Menschen unterwerfende technische Ratio sowie das Artefakt, insofern es diese Ratio gegenüber dem Menschen durchsetzt. Menschliches Handeln verliert hier mit Intentionalität und Freiheit seine wichtigsten Alleinstellungsmerkmale, vom Handeln der technischen Apparate wird es ununterscheidbar.

Die Möglichkeit, Handlungen gerade in diesem Sinne zu konzeptualisieren, wurde bekanntlich um das Ende des 20. Jahrhunderts intensiv reflektiert, verbunden mit einer klaren Absage an Konzepte, die eine Herrschaft der Technik über den

88 Gehlen, Die Technik in der Sichtweise der Anthropologie (1986 [1953]), S. 102.

89 Adorno, Nicht anklopfen (1996 [1951]), S. 44.

90 Gehlen verabschiedet den Begriff von Intentionalität gemeinsam mit der Trennung von Körper und Geist, um sich dem Begriff der Handlung zuzuwenden. Vgl. Gehlen, Zur Geschichte der Anthropologie (1986 [1957]), S. 18.

91 Adorno, Nicht anklopfen (1996 [1951]), S. 44.

92 Ebd. 
Menschen und vice versa implizieren; einer Absage, wie es scheint, an ideologiekritische Standpunkte überhaupt. ${ }^{93}$ Keiner von den beiden in eine Handlung involvierten Parteien bzw. >Aktanten<, so Bruno Latour als Hauptvertreter der AkteurNetzwerk-Theorie, handle unabhängig vom anderen, keinem sei ein genuines Telos eingeschrieben. Handlungsziele sowie Handlungen entstünden erst in der >Vermittlungく, der >Übersetzungく zwischen Mensch und Artefakt. ${ }^{94}$ Die aus ihnen gebildeten Hybride wären in der Moderne im Vormarsch, de facto omnipräsent, während das kritische Projekt ebendieser Epoche irrigerweise an der sauberen Trennung der Sphären arbeitet. ${ }^{95}$

Nichtmenschliche Aktanten halten nach Latour >Skripts (bzw. >Propositionen<, >Handlungsprogramme`) bereit, die sie dem Gegenüber anbieten; das Gegenüber reagiert darauf. ${ }^{96}$ Außerhalb solcher Verkettungen - >Versammlungen $<$ - sind weder Handlungen noch ihre Elemente sinnvoll zu verstehen: »Niemand hat je reine Techniken gesehen - und niemand je reine Menschen ", so Latour. $^{97}$

Menschlichkeit, die nur denkbar ist im Verbund mit Dinglichem (ganz im Sinne Gehlens ${ }^{98}$ ) - damit ist natürlich jenen, die Menschliches von Dinglichem fein säuberlich trennen wollen und alles andere als »skandalöse Verletzung natürlicher Schranken «99 sehen, der Kampf angesagt. Die Opposition von >Menschen ${ }^{9}$ und >Dingen sei nichts als die >modernistische Übereinkunft< der Antifetischisten, eine

93 Zu den Abstrichen, die eine solche Position aus Perspektive der Kritischen Theorie erfordert, vgl. etwa Gransee, Über Hybridproduktionen und Vermittlungen (2003). Zusammenfassend zur nicht-menschlichen Handlungsfähigkeit bei Latour siehe auch Greif, Wer spricht im Parlament der Dinge? (2005), v.a. S. 52-56.

Vgl. etwa Latour, Die Hoffnung der Pandora (2000 [1999]), S. 216-219, 381.

95 Vgl. ders., Wir sind nie modern gewesen (2008 [1991]).

96 Vgl. etwa ders., Die Hoffnung der Pandora (2000 [1999]), S. 375, 379; ders., Ein Türschließer streikt (1996 [1993]), S. 68; Akrich u. Latour, A Summary of a Convenient Vocabulary for the Semiotics of Human and Nonhuman Assemblies (1992), S. 261.

97 Und weiter: »Für Dinge und Menschen gibt es nur eine einzige Syntax und eine einzige Semantik. Anders als es uns die Befürchtungen der Moralisten nahelegen, können wir nicht bestimmte Wörter aus diesem langen Satz herausschneiden, ohne damit ebenfalls das herauszuschneiden, was unsere Menschlichkeit bildet.« Latour, Porträt von Gaston Lagaffe als Technikphilosoph (1996 [1993]), S. 21 u. 26. In der Überzeugung übrigens, dass Humanwissenschaft ohne Einbezug der Dingwelt nicht mehr denkbar ist, findet sich eine Parallele zu Günther Anders, der - freilich aus technikkritischer Perspektive-der Sozialpsychologie, wolle sie wirklich ihren Anspruch erfüllen, eine »Dingpsychologie« ans Herz legt. Vgl. Anders, Die Antiquiertheit des Menschen, Bd. II (1980), S. 261, vgl. S. 58ff. Vgl. Lütkehaus, »Desiderat: Dingpsychologie« (2002).

»Wir geben durchaus zu, dass die Technologien die Fortsetzungen unserer Organe bilden.« - Latour, Das Dilemma eines Sicherheitsgurtes (1996 [1993]), S. 35.

Ders., Ein Türschließer streikt (1996 [1993]), S. 72. 
obsolete Ideologie von sozialer Reinheit und menschlicher Souveränität. ${ }^{100}$ Nachdem ein nicht von Artefakten bestimmter Mensch nicht vorstellbar ist (und umgekehrt), könne es dieser Position zufolge nicht darum gehen, die Bindungen an die Dinge aufzuheben (das wäre eine »barbarische Tortur ${ }^{101}$ ), sondern für gute anstelle von schlechten Bindungen zu sorgen, "those attachements that save from those that kill zu unterscheiden. ${ }^{102}$ Das ist umso wichtiger, als die Kontrolle, die der Mensch über die Artefakte hat, begrenzt ist: »to launch a cascade of irreversible events, yes, to be master of his tools, no. ${ }^{103}$

Alles, was bisher zum Thema Unterwerfung, Angleichung, Verschmelzung gesagt wurde, was also im Kern eine Hybridisierung von Handlung und Intentionalität durch ihre Aufteilung auf Menschen wie Dinge trägt, findet sich hier expliziert. Auch Latour befasst sich mit der selbstschließenden Tür, aber anders als bei Adorno in Form einer euphorischen Aufforderung, das Wissen um die dinglichen Aktanten und ihre Rolle im vermeintlich rein menschlichen Handeln zuzulassen, im Fall der Türen also anzuerkennen, wie sie uns die Last einer Handlung (des Türschließens) und zugleich die Last der Moral (des Willens, die Türe zu schließen) abnehmen. ${ }^{104}$

Dieses Modell lässt sich nun an jeder denkbaren Handlung illustrieren, auch an den zahllosen Verrichtungen Schneiders im Gesäuse und wohl an den Verrichtungen jeder Figur jedes Buches. Eine so umfassende Anwendbarkeit würde die Sache natürlich uninteressant machen, wäre da nicht der Aspekt, dass - Latour zufolge - Handlungen nicht nur im Verbund mit Dingen ausgeführt, sondern von diesen tatsächlich verändert werden: Nicht nur muss das Werkzeug dem Zweck entsprechend gewählt werden, es beeinflusst den Zweck; Artefakte sind keine bloßen Mittel, sondern »unberechenbare Vermittler ${ }^{105}$. Dieser Aspekt der Unberechenbarkeit liegt in jeder >Übersetzung als »einer Verbindung, die vorher nicht da war und die beiden ursprünglichen Elemente oder Agenten in bestimmtem Maße modifiziert «. ${ }^{106}$

Bietet nun Über Raben ein solches Moment des Unberechenbaren? Werden hier Handlungen und Intentionen durch ihre Werkzeuge irgendwo nicht nur ermöglicht, sondern auch beeinflusst, verändert? Durchaus nehmen die Dinge

"Die Menschen sind nicht mehr unter sich. Wir haben schon zu viele Handlungen an andere Aktanten delegiert, die nun unsere menschliche Existenz teilen. Diese Delegation hat sich so weit entwickelt, daß ein Programm des Anti-Fetischismus nur in eine nichtmenschliche Welt führen könnte, eine verlorene, phantasmagorische Welt vor der Vermittlung der Artefakte.« - Ders., Die Hoffnung der Pandora (2000 [1999]), S. $231 f$.

101 Ders., Porträt von Gaston Lagaffe als Technikphilosoph (1996 [1993]), S. 27.

102 Ders., Factures/fractures (1999), passim u. S. 30.

103 Ders., On the Modern Cult of the Factish Cods (2010 [1996]), S. 65

104 Vgl. etwa ders., Ein Türschließer streikt (1996 [1993]), S. 68.

105 Ders., Über technische Vermittlung (1998), S. 65.

106 Vgl. ders., Die Hoffnung der Pandora (2000 [1999]), S. 217f. 
in der Wahrnehmung des Protagonisten emotionale Qualitäten an. Ziemlich ausgeschlossen ist aber, dass hier auf Textebene plötzlich eine Art magischer Realismus Einzug hält (da hätte man doch allerhand überlesen). Zugleich muss eingewendet werden, dass ebenso, wie Latours Erkundungen des Alltags (neben dem Türschließer etwa der Temposchwelle, des Sicherheitsgurtes, des HotelSchlüsselanhängers) nicht-menschliches Agieren erst im Rahmen von behutsamen Vivisektionen offenlegen, auch im Text nur subtile Hinweise zu erwarten sind. Die Beziehung zwischen Mensch und Technik in Über Raben unter Latours Prämissen $\mathrm{zu}$ betrachten, bietet dabei die Möglichkeit, gerade inkriminierende Handlungen in ihren kleinsten Details zu erkunden - und womöglich einen Aspekt des technikkritischen Verdikts zu modifizieren.

In einer Passage, die sich über sechs Seiten erstreckt, beobachtet man den Protagonisten dabei, wie er einen vermeintlichen Verfolger entdeckt und wie die herangezogenen technischen Hilfsmittel den Blick auf diesen Mann beeinflussen:

Als er den Blick senkte, sah er den Mann. Er glaubte erst an ein Blendungsphänomen mit negativem Nachbild der Sonne und rieb sich die Augen. Der schwarze Punkt blieb bestehen, tief unten, inmitten des weiten Kars, das sich in südwestlicher Richtung zwischen Hochtor und Festkogel erstreckte. Er legte den Schokoriegel zur Seite und schaute genauer hin. Der Punkt bewegte sich aufwärts, langsam und abgehackt zwar, aber er bewegte sich. Er griff zum Fernglas.

[...]

Es war ein Mann mit dunkelblauem Stirnband, grauem Pullover und violettem Rucksack. Der Statur nach war es am ehesten Widmann oder Prechtl. Ungewohnte Kleidung konnte die Menschen allerdings völlig verändern. Er suchte die Umgebung sorgfältig ab, vor allem die Waldränder. Er fand niemanden sonst. (ÜR 158f.)

Zuerst - ohne optische Hilfsmittel - ist nicht einmal die Existenz des Mannes sicher; durch das Fernglas wird der Punkt zum Mann, Kleidungs- und Ausrüstungsgegenstände bekommen Farbe. Identifizieren lässt sich der Mann jedoch nicht, die erkennbare Statur gibt dem paranoiden Augenpaar nur ungefähre Hinweise. Der Protagonist greift also der stärkeren Vergrößerung wegen (die an der nun zunehmenden Zahl an sichtbaren Details erkennbar wird) zu seinem Gewehr und blickt durch das Zielfernrohr.

Gewehre gehören zwar nicht generell zur Ausrüstung des Alpinisten, sehr wohl aber zur Ausrüstung dieses speziellen Alpinisten. Das Weatherby des Protagonisten kommt bis zu den - hier vorausgesetzten - Morden nur am Schießstand zum Einsatz; es handelt sich dabei (der niedrige Preis lässt annehmen, dass Schneider sich für das Modell >Vanguard entschieden hat) nicht etwa um ein Kleinkalibergewehr, und bei der Munition nicht um die für das sportliche Schießen geeigneten rückstoß- und leistungsschwachen Kleinkaliberpatronen. Das Weatherby wurde 
ursprünglich für die Jagd entwickelt und ist mit seinem Verschlusssystem für die leistungsstarke Magnum-Munition gebaut. Österreichische Schießstände sind auf Distanzen von 100 bis 300 Metern ausgelegt, auf die diese - zudem sehr teure Art der Munition keinen nennenswerten Vorteil bietet. Hauptanwendungsgebiet der $7 \mathrm{~mm}$ Magnum, die Schneider verwendet, sind Jagden auf große Distanz und verhältnismäßig großes Wild (Hirsch und Sau). ${ }^{107}$ Die Wahl des Gewehrs unterliegt bei Schneider also keineswegs den Beweggründen des Sportschützen; man darf zumindest vermuten, dass neben der Präzision der Waffe auch andere Motive eine Rolle beim Kauf spielen.

»Er zog das Gewehr aus dem Rucksack und legte an«, lautet die obige Passage weiter. Die Handlung, die nun erwartet wird, ist: Zielen, möglicherweise Schießen. Stattdessen handelt es sich um einen erneuten Beobachtungsvorgang:

Durch das Tasco sah er, dass der Mann eine runde Gletscherbrille trug und rote Tourenschier benutzte. Obwohl die Route gespurt war, schien der Mann sich zu plagen. Vor allem bei den Spitzkehren hatte er eindeutig Schwierigkeiten. Er stellte den einen Schi zu wenig weit aus und brachte den anderen dann kaum um die Ecke. Ab und zu trank der Mann aus einer Feldflasche, die er am Gürtel hängen hatte, ab und zu blieb er auch einfach stehen und schaute in die Cegend. Er war offensichtlich länger und hagerer, als er ursprünglich gewirkt hatte, und trug einen schütteren Vollbart. [...] Der Mann hatte ein blaues OrtovoxVerschüttetensuchgerät umgeschnallt, das war deutlich zu sehen. (ÜR 159f.)

Ungerichtetes Erblicken (»sah er den Mann«) und zielorientierter Blick (»suchte [...] sorgfältig«) wechseln einander in diesen Passagen mehrmals ab. Das TascoZielfernrohr ist nicht nur ein Ziel-, sondern zugleich immer auch ein Sehbehelf; was auch immer man vorhat (etwa schießen), es liefert ein Bild mit allen Details. Mit ihm lassen sich Einzelheiten der Gletscherbrille, des Gehstils und der übrigen Ausrüstung, sogar die Marke des Lawinensuchgeräts feststellen; außerdem die Identität des Mannes, insofern zumindest, als er niemand von denen ist, die der Ich-Erzähler unter seinen Verfolgern vermutet. Bis hierher ist nicht eindeutig zu klären, ob Schneider zielen und vielleicht schießen will und dabei nur unbeabsichtigt auch sieht; oder ob er nur genauer sehen will und dabei unbeabsichtigt zielt. Solange das Zielfernrohr montiert ist, legt das technische >Skript fest: SehenWollen ist immer auch Zielen-Müssen. Nun lädt er die Waffe, was seine Intention offenlegt (ÜR 160). Aber schon bevor er auf den Nacken des Mannes einstellt (ÜR 163), nimmt die Waffe den mortifizierenden Akt visuell vorweg:

107 Vgl. Lampel u. Mahrhold, Waffenlexikon (1994), daraus v.a. die Stichworte »]agdliches Schießen«, »Magnum« und »Kaliber- und Patronenwahl bei Büchsläufen«, S. 183-191, 196f., 239. 
Das Knie. Der Außenknöchel, darüber die Kunststoffschale eines Schitourenschuhs. Der rechte Ellbogen. Die haarfreie Stelle hinter dem Ohr. Ein Punkt neben der Spitze des linken Schulterblattes, zwei Fingerbreit zur Mitte hin. (ÜR 162)

Der Apparat verändert das Objekt im Fokus, und nicht zu dessen Vorteil. Mit den Details, die der Text referiert, wird bessere Sichtbarkeit illustriert und im selben Atemzug das Objekt fragmentiert. An die Stelle des Mannes treten sein rechter Ellbogen, der Tourenschuh. Das bildet sich auch sprachlich ab: Andere körperliche Merkmale, die dünnen Beine etwa und die Kniescheiben (ÜR 162), sind noch Teile vollständiger Syntagmen, die später genannten Details - »Das Knie. Der Außenknöchel « und so fort - haben sprachlich die Form von Nominalphrasen. Bildlich suggeriert die Aufeinanderfolge vereinzelter Details abgetrennte Teile, die Zergliederung eines Organismus. Die Sichtweise, die das Zielfernrohr erlaubt, imitiert damit visuell den Zweck des Gewehrs, die Verwandlung vom Lebendigen ins Tote.

Im dargestellten Blick treffen die Intentionen zweier Aktanten aufeinander, ${ }^{108}$ des Deutschlehrers und seines Zielfernrohrs; solange er sehen will, muss er zugleich zielen, sobald er zielt, nimmt die Waffe das Ergebnis vorweg. Im Vorgang des Sehens ist die Mortifikation des betrachteten Objekts impliziert, sowohl konkret hinsichtlich des unwissentlich in Lebensgefahr schwebenden Tourengängers als auch hinsichtlich der zerstückelnden Tendenz jeder Vergrößerung. »Mikroskope und Fernröhre«, ließe sich mit Goethe feststellen, »verwirren eigentlich den reinen Menschensinn ${ }^{109}$ (wohingegen es nach Latour ja keine >reinen Menschen gibt, s.o.).

»Wer ist nun also der Akteur in meiner kleinen Geschichte, die Waffe oder der Mörder?« - so Latour in einer Passage über Schusswaffengebrauch. Der >Symmetrie< seines Modells entsprechend fährt er fort: »Mit der Waffe in der Hand bist $\mathrm{du}$ jemand anderes, und auch die Waffe ist in deiner Hand nicht mehr dieselbe." Es sei ein Irrtum, anzunehmen, »das psychische Vermögen der Menschen sei ein für allemal festgelegt. «110 Die Frage, ob denn Schneider auch ohne seine Waffe zum Mörder geworden wäre - oder ob er eine andere Methode als das Erschießen gewählt hätte - ist irrelevant. Der Hintergrund, das Konzept nicht-menschlicher

108 Die Konzentration auf diesen kleinen Kreis an Aktanten ist natürlich eine Vereinfachung, bedenkt man die Vielzahl an Personen, Institutionen und Technologien, die in diesem Ceschehen verschränkt sind; nicht das Flugzeug fliegt, sondern die gesamte Fluglinie, wie Latour diesbezüglich anmerkt - der nichtsdestoweniger zahlreiche Beispiele so eng abgezirkelter >Versammlungen bringt, nicht zuletzt in illustrativer Absicht. Ebenso ist natürlich Adornos Türschließer-Beispiel zugleich als Detailbeobachtung und als Exempel zu lesen. 
Agency, das das close reading der Passage mit sich bringt, eröffnet nun aber die Option, dass auch die Waffe Einfluss auf die Handlung hat. ${ }^{111}$

Eine der Erinnerungen des Protagonisten gilt einem Kletterkollegen und dessen Aussage, »das mit dem Schießen verstehe er nicht, schon gar nicht bei jemandem, der beruflich ständig mit Kindern zu tun habe. `Genau deswegen<, hatte er ihm geantwortet, und das war es auch schon gewesen."(ÜR 163) - Hier unterbleibt die Erklärung, aus der rekonstruierten Vorgeschichte des Textes lässt sich Schneiders Wahl seines Hobbys jedoch plausibel machen: Ein Lehrer erkennt ein innerfamiliäres Problem, kann darauf aber innerhalb der gesetzlichen Richtlinien (vielleicht nicht zum ersten Mal) nicht angemessen reagieren. Die tendenzielle Ohnmacht Außenstehender, wenn es um Delikte innerhalb der Familie und ihre Nachweisbarkeit geht, verhandeln Hochgatterers Texte oft (siehe etwa Das Milchhautkind, Die Süße des Lebens, Das Matratzenhaus). In Schneiders Fall jedoch ist dieser Zwiespalt in den technischen Mitteln aufgehoben: Ein Gewehr mit einem Zielfernrohr vereint Wahrnehmungs- und Handlungsmöglichkeit in sich. Ganz anders als in Schneiders Erfahrung als Lehrer bedeutet Sehen-Müssen hier - qua Skript auch Handeln-Können. Der Schießsport als Ventil für den Alltag bildet das komplementäre Gegenbild der beruflichen Situation, und man könnte folgern: Gerade dass er das so perfekt tut, führt zur Kontamination der beiden Sphären, der Überführung des Gewehrs von einer in die andere. Indem Freizeit hier das Subjekt nicht für, sondern gegen die gesellschaftlich sanktionierte Rolle zurichtet, ist der Kritischen Theorie widersprochen; wobei freilich die Rolle der Technik zum Scharnier der Katastrophe wird.

Der irritierenden Schlussfolgerung Latours, in einem solchen Zusammenhang nicht nur den Ablauf, sondern tatsächlich auch die Verantwortung für den abgegebenen Schuss zwischen Mensch und Artefakt aufzuteilen, ${ }^{112}$ muss man jedoch mit Blick auf Über Raben nicht folgen: schlicht deshalb, weil Schuld im ganzen Text nie thematisiert wird (das bleibt den Leserinnen und Lesern überlassen). Dargestellt werden ausschließlich Verhältnisse von Ursache und Wirkung. Dass sich die Gemengelage aus Missbrauch und Selbstjustiz in Über Raben auf irgend eine gefällige Weise auflösen lässt, war nicht zu erwarten. Wenn überhaupt, kann nur die Rede sein von einer Auflösung in eine unterkühlte Kette auseinander hervorgehender Ursachen. Während (bzw. gerade indem) der Text ja die Auslöser seines Plots - die

111 Eine Anmerkung zum Zielfernrohr: Im Text wird es als »Supersnider « angeführt - recte: >Tasco Super Sniper<. Möglicherweise ist das ein Tippfehler. Allerdings enthält ihn auch noch die Taschenbuchausgabe von 2009, sodass es sich möglicherweise um ein Wortspiel mit ssnider<->schneider handelt.

112 Latour, Die Hoffnung der Pandora (2000 [1999]), S. 218: »Weder Menschen noch Waffen töten. Vielmehr muß die Verantwortung für ein Handeln unter den verschiedenen Akteuren verteilt werden.« 
Toten - unterschlägt, besteht er auf der Logik, die aus ihnen wirkt, auf der Kausalkette, die aus ihnen hervorgeht - und in der die Artefakte mit ihren Skripten und Defekten eine tragende Rolle spielen.

\section{Schluss}

Zeit für ein Resümee: Die warenästhetischen und anderen landläufigen Fetischismen, die Schneiders Verhältnis zu den Dingen schon auf den ersten Blick kennzeichnen, provozierten unseren Blick auf seine Ausrüstung und ihren vermeintlich tadellosen Werkzeugcharakter. Ein Blick in den alpinistischen Diskurs zur Technik am Berg sowie zum Ort der Handlung - der Route im Gesäuse - macht leicht erklärlich, wieso der Aspekt der technischen Verrichtung am Berg in diesem Roman so stark prononciert ist: weil Technikeinsatz im Diskurs des Alpinismus ein Kriegsschauplatz ist.

Schneiders Haltung zu diesem Konflikt zeigt einerseits, dass der Diskurs zur Technik den Alpinismus nicht nur beschreibt, sondern längst Teil davon ist: Der Berg wird als ein auch durch diese Texte geformtes kulturelles Artefakt wahrgenommen. Der reflektierte, zitathafte Umgang des Protagonisten mit der Technik, die in diesem Diskurs verhandelt wird, und seine entspannte, pragmatische bis affirmative Haltung zu dieser entsprechen einer historischen Periode, in der der Konflikt an Brisanz verloren hat.

Andererseits widerspricht die Logik des Textes einem affirmativen Verhältnis zur Technik krass: Der Defekt zeigt sich letztlich als ihr bestimmendes Prinzip. Die Konfrontation technikaffirmativer und technikkritischer Tendenzen im Text sowie ihrer jeweiligen Kontexte - schlaglichtartig: der philosophischen Anthropologie und der Kritischen Theorie - macht zudem deutlich, wie Technik als Ergänzung des >Mängelwesens` Mensch auch dessen Unterwerfung unter eine technische Ratio und dessen Angleichung an den leblosen Mechanismus betreibt. Der Protagonist des Romans illustriert diese Angleichung nicht nur, sondern führt sie konsequent zu Ende. Indem er damit nicht mehr souveränes Subjekt ist, sondern zum Teil eines organisch wie mechanisch gebildeten Regelkreises wird, agiert die Logik der Dinge in seine Handlungen hinein.

Der Schluss des Romans ist schließlich einer, der konsequent sowohl aus der menschlichen wie der technischen Dramaturgie des Textes - oder genauer: aus der Verschränkung beider - folgt. Anders als etwa in der Kurzen Geschichte vom Fliegenfischen findet sich kein relativierender Blick auf die Skandale des Fetischismus. Das Urteil der Gesäuse-Kapitel von Über Raben übertrifft in seinem Pessimismus noch die Verdikte, die im kulturellen Archiv zur Annäherung an, zur Unterwerfung unter die Technik, zur Übertretung der geheiligten Grenze von Subjekt und Objekt, letztlich zu dem jedem Werkzeuggebrauch inhärenten Fetischismus vielfach vorliegen. Das technische Artefakt beschleunigt und katalysiert eine letztlich fatale 
160 Üble Dinge. Materialität und Fetischismus in der Prosa Paulus Hochgatterers

Handlungskette - und ist dabei mehr als bloß passiver dramatischer Erfüllungsgehilfe. 